\title{
Equilibrium Asset Returns in Financial Markets
}

\author{
Dilip B. Madan \\ Robert H. Smith School of Business \\ University of Maryland \\ College Park, MD 20742. \\ Wim Schoutens \\ Department of Mathematics \\ K.U. Leuven \\ Leuven, Belgium.
}

March 7, 2018

\begin{abstract}
Return distributions in the class of pure jump limit laws are observed to reflect numerous asymmetries between the upward and downward motions of asset prices. The return distributions are modeled by self decomposable parametric laws with all parameters continuously responding to each other. Fixed points of the response functions define equilibrium distributions. The equilibrium distributions that can arise in practice are constrained by the level of return acceptability they may attain. As a consequence expected returns are the cost of purchasing the the negative of the centered return. They are then equal to the ask price in two price economies, for the centered and negated return. The asymmetries studied include differences in scale, speed, power variation, excitation and cross excitation.

Keywords: Bilateral Gamma, Power Variation, Support Vector Machine Regression, Acceptability Index

JEL Classification: G11, G12, G13.

\section{Introduction}

The classical perspective on asset returns recognizes that the returns are risky and seeks to explain the expected return or the mean of the return distribution as compensation for the inherent risk. Centered return distributions are purely composed of risk, with the latter being typically measured by the variance. Portfolio considerations are seen as assisting variance reduction via diversification with mean returns reflecting the compensation for systematic or undiversifiable risk as represented by the return covariance with systematic factors. The return to the systematic factors constitutes their risk premium. These are the central
\end{abstract}


themes of the capital asset pricing models of Sharpe (1964), Lintner (1965), Mossin (1966) and the subsequent development of the Ross (1976) arbitrage pricing theory. They are effectively summarized in numerous text books on the subject including Cochrane (2001), Duffie (2001), and Skiadas (2009).

But why are returns risky? Clearly, they need not be if the return expected was just the interest rate or the so called risk free rate. Expected returns that differ significantly from this risk free rate on either side will attract investors and or speculators seeking to exploit any momentum or to benefit from an anticipated mean reversion. The result of these activities will create the risk and the resulting variance. Hence not only are expected returns responding to the risk, however measured, but the risk is also responding to the expected return. Studies in this direction include Schwert (1989) and Engle, Ghysels and Sohn (2008). These interactions must result in some stable, acceptable, temporally equilibrium return distributions. In this regard, we concur with the view expressed in Weitzman (2007) of an essentially nonergodic situation with no set of true parameters for the return distributions to converge to. Whatever equilibria one effectively solves for are at best transitory.

In principle such equilibrium return distributions could be studied at the level of multivariate distributions or return generating processes. Similar assets or stocks of companies in comparable lines of business may not have independent return distributions and the joint distribution would be expected to reflect some correlation driven by market participants looking at them in tandem. Unusual movements in one may trigger corrective actions in another. The longer the return horizon the greater are the possibilities for developing the correlations. This phenomenon known as the Epps effect has been known since Epps (1979) and is further documented in Bonanno, Lillo and Mantegna (2001). Over short horizons correlations and portfolio considerations are less relevant. Most traders holding positions for a short span of time are not evaluating or exploiting cross asset return dependencies or diversification benefits, but rather seeking out short lived mispricings one asset and one moment at a time. The variance is being monitored by the short span of time for which positions are being held.

Attention is therefore focused here, not on equilibrium mean returns given risk, but on equilibrium levels for the entire return distribution taken one asset at a time for the shorter horizons. For the physical return distribution the object of study is the daily return distribution. Risk neutrally we consider mainly the maturities below a month. In both cases a parametric class of distributional possibilities is first adopted as the default. Within the chosen parametric class the equilibrium return distribution is identified on determining equilibrium levels for the parameters. For this purpose a one to one mapping between certain moments and the parameters is employed to infer equilibrium parameters from corresponding equilibrium levels for the selected moments. The moments are seen as responding to each other and the equilibrium is given by the fixed point for this response mapping. Such an equilibrium construction was made in Madan and Schoutens (2018) and it is here generalized and extended to a wider class of processes underlying the daily returns. In this way one may construct equilibrium daily return distributions for each asset each day under the physical 
process for returns. Risk neutrally one seeks to construct equilibrium volatility surfaces each day for each underlying asset on which options are traded.

Ultimately one develops a time series of estimated parameters for the return distribution in the default parametric class and a time series of the estimated equilibrium parameters. Lead lag relationships between these may be investigated to identify regions of parametric momentum when the equilibrium levels lag the estimated levels and the regions of parametric mean reversion when the estimated levels lag the equilibrium ones. It may be possible for both phenomena to occur simultaneously for different parameters. Hence one may have mean reversion in drifts with momentum for volatility or the other way around. A study of these issues is a topic for future research. The objective here is to first identify the equilibrium levels.

Critical to such an exercise are the default parametric class of distributions. Clearly the class must be capable of fitting the data but yet not be so wide as to permit over fitting. Structural restrictions help reduce parameters and avoid over fitting while some parametric richness helps in differentiating possibilities away from imposing too many symmetries that may not be present. In this regard we rely on our experience in fitting return distributions both risk neutrally and in the time series data permitting the parametric richness being sought and on structural theoretical restrictions limiting overfitting.

The theoretical restriction is to employ a limit law for the distribution, on the grounds that the number of price moves involved in unit time, though finite, is quite large. The limit laws arise at infinitely many moves and have been characterized as the self decomposable laws by Lévy (1937) and Khintchine (1938). They are a subclass of the infinitely divisible distributions with a further restriction on the associated Lévy density. Arrival rates of jumps when scaled by the absolute jump size must be decreasing functions of the absolute jump size. The self decomposable laws also have provably unimodal distributions (Sato (1999)) and thereby refrain from overfitting a multiple of modes.

There are asymmetries between the way market prices rise and how they fall. It is often said that markets take the escalator up and the elevator down. We cite two references from the web Such references may be found on the web and we cite two examples http://allstarcharts.com/escalator-up-and-elevator-down/, and http://seekingalpha.com/article/3746396-market-stars-ride-escalarot-elevator. Recently these differences were explored and documented in Madan and Wang (2017), and Madan, Schoutens and Wang (2017). Here they are further expanded upon. We refrain from employing a continuous component in the price process or its logarithm. These processes impose a symmetry between the upward and downward motion and do not permit a split between the two. They are also processes of infinite variation and do not allow for the two processes of upward and downward motion to have an independent existence. Already the use of limit laws stretches reality by allowing for infinitely many moves. Infinite variation is yet another stretch away from reality. Processes in continuous time are employed for their analytical convenience recognizing that as a description of reality they are not really admissible. Physical time, eventually marked by precise time stamps, is at best discrete. Hence we work primarily with pure jump 
processes of finite variation and shall document the differences to be found physically and risk neutrally in the separate processes for the upward and downward motion.

A particularly simple pure jump process with a self decomposable law at unit time is given by the variance gamma model of Madan and Seneta (1990) and Madan, Carr and Chang (1998). The jump arrival rate function when scaled by the absolute jump size is just a negative exponential of the absolute jump size, a clearly decreasing positive function. It permits a separation of the upward and downward motion, with both processes being gamma processes with separate scale parameters and the same shape or speed parameters. Madan and Wang (2017), Madan, Schoutens and Wang (2017) document that identical sppeds on the two sides may be overly restrictive and they employ the bilateral gamma process of Küchler and Tappe (2008). This is a difference of two gamma processes with their own speed and scale parameters. It is observed that the upward motion has a higher speed and lower scale parameter when compared to the same for the downward motion.

The first parametric class employed in the construction of equilibrium return distributions is the bilateral gamma. However, we go on to address other asymmetries beyond scale and speed. Beyond speed and scale we go on to consider asymmetries in power variation. This is the smallest power $\alpha$ such that the $\alpha$ power variation is finite. Given that we work with semimartingales with finite quadratic variation the smallest power with a finite power variation is less than 2. For a process of independent and identically distributed increments three asymmetries are documented, scale, speed and the power variation.

One may go futher in the context of a Markov process to document how the parameters of local motion respond to what has occured to date. For diffusions this takes us to local volatility models when the volatility is made to depend on the level of the process and or calendar time. In the interests of parsimony and with a focus on local dynamics we supress any dependence on calendar time and work with time homogeneous Markov processes. This would suggest making all of speed, scale and power variation functions of level of the process. In the interests of pursuing and documenting asymmetries we consider functions of not the price level or process level, but the aggregated positive and negative moves so far. The dependence could be in all parameters and nonlinear at that. The resulting structure is not only analytically intractable but also numerically intractable. However we may incorporate as numerically tractable the linear dependence of speed on the aggregated positive and negative moves. The infinitesimal generator is then linear in state variables permitting an exponential affine solution for the characteristic function (Duffie, Filipovic and Schachermayer (2003)) that leads to ordinary differential equations for the coefficients. The solution for the characteristic function then requires the numerical solution of thousands of ordinary differential equations to get the characteristic function. Fourier inversion then delivers the density and or the option prices. Calibration is possible in a matter of a few hours on current computing capacities. This linear dependence of speed separately on the aggregated positive and negative moves we term excitation and cross excitation depending on whether we are 
referring to the speed of positive moves responding to the aggregated positive and negative moves respectively. Similarly for the speed of the negative moves. The five asymmetries studied and reported on in the paper will then be scale, speed, power variation, excitation and cross excitation. For excitation and cross excitation we report the results of estimations conducted but equilibrium constructions are not possible given the computational costs of single estimations.

Estimations are conducted both for the physical daily return distribution and risk neutrally at the shorter maturities. In both cases we identify the structure of jump arrival rates. The arrival rates may risk neutrally be lifted by running time faster and they may also be altered by changing the scale. In continuous time a measure change may change scale but not speed, but as the continuous time limit is not the reality but just a model, we permit changes in speed as well. Beyond speed and scale we consider the power variation. Again this parameter may not be changed by an equivalent change of probability in continuous time as it is embedded in the paths and may be recovered from them. However, we entertain such changes as well.

Given the classically special attention focused on explaining mean returns we turn to explaining the equilibrium mean returns of our equilibrium distributions. Given the absence of portfolio considerations at the level of daily returns the mean returns are explained by the implications for avoiding excessively good deals. The level of a deal is measured by the acceptability index of the equilibrium return distribution as constructed in Cherny and Madan (2009). Mean returns may then be related to the risk of a return distribution as measured by the ask price for the centered and negated return. Asset pricing implications along these lines are formulated and tested.

The outline of the rest of the paper is as follows. Section 2 introduces models for the five asymmetries being reported on and the details for the characteristic function constructions that are employed in estimating daily return distributions and calibrating volatility surfaces. Section 3 reports on the first three asymmetries of scale, speed and power variation in the physical process. The risk neutral counterparts are taken up in Section 4. For both constructions equilibrium return distribution and volatility surfaces are reported on in Section 5 and 6 respectively. Section 7 takes up asset pricing implications and their tests. Sections 8 reports on the physical and risk neutral estimation of excitation and cross excitation respectively. Section 9 concludes.

\section{Five asymmetries of motion}

The first two asymmetries of motion are those of speed and scale and these are captured in the bilateral gamma process. Let $\gamma(t), t>0$ be a path of the standard gamma process with unit scale and speed. For fixed $t$, the random variable $G_{t}=\gamma(t)$ has a gamma density $f(g)$ given by

$$
f(g)=\frac{g^{t-1} e^{-g}}{\Gamma(t)} .
$$


The gamma process is an infinitely divisible pure jump, increasing process with arrival rates of jumps of size $x$ given by the arrival rate function $k_{\gamma}(x)$ where

$$
k_{\gamma}(x)=\frac{e^{-x}}{x}, x>0
$$

The aggregate arrival rate is infinite and the process is one of infinite activity with infinity many arrivals in any time interval. The characteristic function $\phi_{G}(u)$ is given by

$$
\begin{aligned}
\phi_{G t}(u) & =E\left[e^{i u G_{t}}\right] \\
& =\left(\frac{1}{1-i u}\right)^{t} .
\end{aligned}
$$

The bilateral gamma process $X(t), t>0$ has four parameters $b_{p}, c_{p}, b_{n}$, and $c_{n}$ that are the scale and speed parameters for the upward and downward motion respectively. For two independent standard gamma processes $\gamma_{p}(t), \gamma_{n}(t), t>0$ on has

$$
X(t)=b_{p} \gamma_{p}\left(c_{p} t\right)-b_{n} \gamma_{n}\left(c_{n} t\right) .
$$

The characteristic function for the bilateral gamma is given by

$$
\begin{aligned}
\phi_{X}(u, t) & =E[\exp (i u X(t))] \\
& =\left(\frac{1}{1-i u b_{p}}\right)^{c_{p} t}\left(\frac{1}{1+i u b_{n}}\right)^{c_{n} t} .
\end{aligned}
$$

The arrival rate of jumps $x \neq 0$ is given by $k_{X}(x)$ where

$$
k_{X}(x)=c_{p} \frac{\exp \left(-\frac{x}{b_{p}}\right)}{x} \mathbf{1}_{x>0}+c_{n} \frac{\exp \left(-\frac{|x|}{b_{n}}\right)}{|x|} \mathbf{1}_{x<0} .
$$

In the special case of identical speeds up and down with $c_{p}=c_{n}=1 / \nu$ one gets the variance gamma process of Madan and Seneta (1990), Madan, Carr and Chang (1998) that may be represented as Brownian motion with drift $\theta$ and volatility $\sigma$ time changed by a gamma process with unit mean rate and variance rate $\nu$. In this case

$$
\begin{aligned}
\theta & =\frac{b_{p}-b_{n}}{\nu} \\
\sigma^{2} & =\frac{2 b_{p} b_{n}}{\nu} .
\end{aligned}
$$

The variance gamma (VG) has an arrival rate function described in Carr, Geman, Madan and Yor (2002) as

$$
k_{V G}(x)=C \frac{\exp (-M x)}{x} \mathbf{1}_{x>0}+c_{n} \frac{\exp (-G|x|)}{|x|} \mathbf{1}_{x<0}
$$


with $C=1 / \nu, G=1 / b_{n}$, and $M=1 / b_{p}$. The variance gamma has a density $f_{V G}(x)$ in closed form given by (See Carr and Madan (2014))

$f_{V G}(x)=\frac{(G M)^{C}}{2^{C-1} \Gamma(C) \sqrt{2 \pi}\left(\frac{G+M}{2}\right)^{C-1 / 2}} \exp \left(\frac{G-M}{2} x\right)|x|^{C-1 / 2} K_{C-1 / 2}\left(\frac{G+M}{2}|x|\right)$,

where $K_{\nu}(x)$ is the modified Bessel functions. The variance gamma may be first estimated to obtain starting values for estimating the bilateral gamma by Fourier inversion of the characteristic function. Madan and Wang (2017) and Madan, Schoutens and Wang (2017) have reported that the upward process has higher speed and lower scale when compared to the downward process.

\subsection{The asymmetry of power variation}

The CGMY model of Carr, Geman, Madan and Yor (2002) permits a differentiation of power variation for the process of upward moves from downward moves if the parameters $C, Y$ are split to allow for separate values on the two sides. We denote this model $P V$ for power variation. The arrival rate function $k_{P V}(x)$ is given by

$$
k_{P V}(x)=c_{p} \frac{\exp \left(-x / b_{p}\right)}{x^{1+y_{p}}} \mathbf{1}_{x>0}+c_{n} \frac{\exp \left(-|x| / b_{n}\right)}{|x|^{1+y_{n}}} \mathbf{1}_{x<0} .
$$

The additional parameters beyond the bilateral gamma are $y_{p}, y_{n}$ and the $\alpha$ power variation on the positive side given by

$$
\int_{0}^{\infty} x^{\alpha} k_{P V}(x) d x<\infty
$$

for all $\alpha>y_{p}$. Similarly for the negative side for $\alpha>y_{n}$ the $\alpha$ power variation is finite. The characteristic function is given by

$$
\phi_{P V}(u)=\frac{c_{p} \Gamma\left(-y_{p}\right)}{b_{p}^{y_{p}}}\left(\left(1-i u b_{p}\right)^{y_{p}}-1\right)+\frac{c_{n} \Gamma\left(-y_{n}\right)}{b_{n}^{y_{n}}}\left(\left(1+i u b_{n}\right)^{y_{n}}-1\right) .
$$

Again we first estimate the variance gamma and use the variance gamma estimates as starting values for the power variation model that is estimated by Fourier inversion of the characteristic function.

For the physical process the drift if any is constructed from the asymmetry in the jump arrival rates and hence the process is one finite variation on both sides with $y_{p}, y_{n}<1$. Risk neutrally one adds drifts determined by the forward price to compensated jump martingales and so one may allow for infinite variation and constructed the two compensated jump martingales for the upward compensated jumps and the downward compensated jumps. We have already commented in the introduction on the possibilities for changing all parameters between the physical and risk neutral estimates. 


\subsection{The asymmetries of excitation and cross excitation}

Let $X_{p}(t), X_{n}(t), t>0$ be the aggregated upward and downward moves to time $t$. More general asymmetries may be modeled by making all the parameters of motion functions of these aggregated entities. However, one may lose tractability for the characteristic function either analytically or numerically in such constructions. Madan (2016) employs continuous time finite state Markov chain approximations to access probability elements in such one dimensional formulations. However, here the Markov dimension is two and such approximations would be more involved. If the infinitesimal generator is kept linear in state variables then the characteristic function is known to be in exponential affine class and some tractability may be possible.

With these considerations in mind consider the two dimensional process $X(t)=\left(X_{p}(t), X_{n}(t)\right) \in \mathbb{R}_{+}^{2}$ with jumps $x_{p}, x_{n}$ and compensator

$$
\begin{aligned}
k\left(X_{p}, X_{n}, x_{p}, x_{n}\right)= & \left(c_{p}+d_{p} X_{p}+e_{p} X_{n}\right) \frac{\exp \left(-\frac{x_{p}}{b_{p}}\right)}{x_{p}} \mathbf{1}_{x_{p}>0, x_{n}=0} \\
& +\left(c_{n}+d_{n} X_{n}+e_{n} X_{p}\right) \frac{\exp \left(-\frac{x_{n}}{b_{n}}\right)}{x_{n}} \mathbf{1}_{x_{n}>0, x_{p}=0}
\end{aligned}
$$

The speed of upward and downward motion is now responsive differentially to the accumulated upward and downward motion to date. Such a model incorporates both excitation and cross excitation for both processes. The coefficients $d_{p}, d_{n}$ calibrate excitation while $e_{p}, e_{n}$ calibrate the cross excitation.

For the characteristic function we wish to evaluate the exponential affine form

$$
\begin{aligned}
& E_{t}\left[\exp \left(i u X_{p}(1)-i u X_{n}(1)\right)\right] \\
= & \phi(t, u) \\
= & \exp \left(a(1-t, u)+b(1-t, u) X_{p}(t)-c(1-t, u) X_{n}(t)\right)
\end{aligned}
$$

The infinitesimal generator is

$\mathcal{A} f\left(X_{p}, X_{n}\right)=\int_{0}^{\infty} \int_{0}^{\infty}\left[f\left(X_{p}+x_{p}, X_{n}+x_{n}\right)-f\left(X_{p}, X_{n}\right)\right] k\left(X_{p}, X_{n}, x_{p}, x_{n}\right) d x_{p} d x_{n}$.

The martingale porperty for $\phi(t, u)$ implies that

$$
\phi_{t}+\mathcal{A} \phi=0
$$

Upon evaluation we get

$$
\begin{array}{ll} 
& -a_{t}-b_{t} X_{p}+c_{t} X_{n}+ \\
& -\left(c_{p}+d_{p} X_{p}+e_{p} X_{n}\right) \ln \left(1-b b_{p}\right) \\
& -\left(c_{n}+d_{n} X_{n}+e_{n} X_{p}\right) \ln \left(1+c b_{n}\right) \\
= & 0 .
\end{array}
$$


The equations for the coefficients of the chacteristic function are

$$
\begin{aligned}
b_{t} & =-d_{p} \ln \left(1-b b_{p}\right)-e_{n} \ln \left(1+c b_{n}\right) \\
c_{t} & =d_{n} \ln \left(1+c b_{n}\right)+e_{p} \ln \left(1-b b_{p}\right) \\
a_{t} & =-c_{p} \ln \left(1-b b_{p}\right)-c_{n} \ln \left(1+c b_{n}\right)
\end{aligned}
$$

Define

$$
\begin{aligned}
y & =b b_{p} \\
w & =c b_{n}
\end{aligned}
$$

to get

$$
\begin{aligned}
y^{\prime} & =-b_{p} d_{p} \ln (1-y)-b_{p} e_{n} \ln (1+w) \\
w^{\prime} & =b_{n} e_{p} \ln (1-y)+b_{n} d_{n} \ln (1+w)
\end{aligned}
$$

This system of nonlinear ordinary differential equations may be solved for $y, w$ subject to the initial conditions

We want to solve for initial conditions

$$
\begin{aligned}
y(0, u) & =i u b_{p} \\
w(0, u) & =i u b_{n} .
\end{aligned}
$$

The function $a(1-t, u)$ follows on numerical integration. The characteristic function may be employed to obtain the density by Fourier inversion.

\subsection{Risk Neutral Considerations}

For the risk neutral calibration of models to data on option prices one needs to formulate the characteristic function for the risk neutral martingale model that is shifted to match the forward price. The martingale process associated with the jump process $X(t)$, with a finite variation arrival rate function $k_{X}(x, t)$, is the compensated jump process $M_{X}(t)$ where

$$
M_{X}(t)=\exp \left(X(t)-\int_{0}^{t} \int_{-\infty}^{\infty}\left(e^{x}-1\right) k_{X}(x, s) d x d s\right) .
$$

For processes with independent increments $X(t)$ the arrival rate function is a deterministic function of the jump size and time and the characteristic function of $M_{X}(t)$

$$
\begin{aligned}
\phi_{M_{X}}(u, t) & =E\left[\exp \left(i u M_{X}(t)\right)\right] \\
& =\phi_{X}(u, t) \exp (i u \omega(t)) \\
\omega(t) & =-\ln \left(\phi_{X}(-i, t)\right)
\end{aligned}
$$


The construction in equation (1) also suffices for processes of infinite variation but the martingale construction takes the form

$$
M_{X}(t)=\exp \left(\int_{0}^{t} b(u) d u+X(t)-\int_{0}^{t} \int_{-\infty}^{\infty}\left(e^{x}-1-x \mathbf{1}_{|x| \leq 1}\right) k_{X}(x, s) d x d s\right)
$$

for a drift function $b(t)$.

The bilateral gamma and power variation processes we work with have self decomposable laws at unit time. They are therefore infinitely divisible and have associated Lévy processes. However, recognizing that Lévy processes do not fit option surfaces across maturity we employ the associated Sato processes as introduced in Carr, Geman, Madan and Yor (2007). The law at time $t$ is obtained by scaling the law at unit time and defining

$$
X(t) \stackrel{(d)}{=} t^{\gamma} X(1)
$$

for a scaling coefficient $\gamma$. The number of parameters for the risk neutral process is thereby increased by one to allow for the scaling coefficient. Sato (1991) shows the existence of an additive process with independent but time inhomogeneous increments that have the marginal distributions at each $t$ consistent with the supposed scaling. The risk neutral processes are such scaled self decomposable processes. The risk neutral stock price process then takes the form

$$
S(t)=F(t) \exp (X(t)+\omega(t))
$$

where $F(t)$ is the forward price for maturity $t$.

These characteristic function constructions suffice for the bilateral gamma and power variation additive risk neutral processes. When we extend to the asymmetries of excitation and cross excitation the drift corrections must be introduced locally in the infinitesimal generator and they lead to changes in the ordinary differential equations that must be solved for the coefficients of the exponential affine form of the characteristic function.

Consider the two dimensional process $X(t)=\left(X_{p}(t), X_{n}(t)\right) \in \mathbb{R}_{+}^{2}$ with jumps $x_{p}, x_{n}$ and compensator

$$
\begin{aligned}
k\left(X_{p}, X_{n}, x_{p}, x_{n}\right)= & \left(c_{p}+d_{p} X_{p}+e_{p} X_{n}\right) \frac{\exp \left(-\frac{x_{p}}{b_{p}}\right)}{x_{p}} \mathbf{1}_{x_{p}>0, x_{n}=0} \\
& +\left(c_{n}+d_{n} X_{n}+e_{n} X_{p}\right) \frac{\exp \left(-\frac{x_{n}}{b_{n}}\right)}{x_{n}} \mathbf{1}_{x_{n}>0, x_{p}=0}
\end{aligned}
$$

For the risk neutral provess we want the exponential of

$$
X_{p}(t)-X_{n}(t)
$$

to be a martingale and hence we need to incorporate an exponential compensation. This leads us to a drift compensation of the form

$$
-g_{p}\left(X_{p}(t), X_{n}(t)\right)-g_{n}\left(X_{p}, X_{n}\right) d t
$$


where

$$
\begin{aligned}
& g_{p}\left(X_{p}, X_{n}\right)=\left(c_{p}+d_{p} X_{p}+e_{p} X_{n}\right) \int_{0}^{\infty}\left(e^{x_{p}}-1\right) \frac{\exp \left(-\frac{x_{p}}{b_{p}}\right)}{x_{p}} d x_{p} \\
& g_{n}\left(X_{p}, X_{n}\right)=\left(c_{n}+d_{n} X_{n}+e_{n} X_{p}\right) \int_{0}^{\infty}\left(e^{-x_{n}}-1\right) \frac{\exp \left(-\frac{x_{n}}{b_{n}}\right)}{x_{n}} d x_{n} \\
& g_{p}\left(X_{p}, X_{n}\right)=-\left(c_{p}+d_{p} X_{p}+e_{p} X_{n}\right) \ln \left(1-b_{p}\right) \\
& g_{n}\left(X_{p}, X_{n}\right)=-\left(c_{n}+d_{n} X_{n}+e_{n} X_{p}\right) \ln \left(1+b_{n}\right)
\end{aligned}
$$

The infinitesimal generator of the 2 dimensional Markov process under consideration is then

$$
\begin{aligned}
\mathcal{A f}\left(X_{p}, X_{n}\right)= & -g_{p}\left(X_{p}, X_{n}\right) f_{X_{p}}-g_{n}\left(X_{p}, X_{n}\right) f_{X_{n}} \\
& +\int_{0}^{\infty} \int_{0}^{\infty}\left[f\left(X_{p}+x_{p}, X_{n}+x_{n}\right)-f\left(X_{p}, X_{n}\right)\right] k\left(X_{p}, X_{n}, x_{p}, x_{n}\right) d x_{p} d x_{n} . \\
g_{p}\left(X_{p}, X_{n}\right)= & -\left(c_{p}+d_{p} X_{p}+e_{p} X_{n}\right) \ln \left(1-b_{p}\right) \\
g_{n}\left(X_{p}, X_{n}\right)= & -\left(c_{n}+d_{n} X_{n}+e_{n} X_{p}\right) \ln \left(1+b_{n}\right)
\end{aligned}
$$

We take the characteristic function in exponential affine form to be

$$
\begin{aligned}
\phi^{T}(t, u) & =E_{t}\left[\exp \left(i u X_{p}(T)-i u X_{n}(T)\right)\right] \\
& =\exp \left(a(T-t, u)+b(T-t, u) X_{p}(t)-c(T-t, u) X_{n}(t)\right) .
\end{aligned}
$$

The martingale condition for the characteristic function then yields the equation

$$
\phi_{t}^{T}+\mathcal{A} \phi^{T}=0 .
$$

Specifically we have

$$
\begin{array}{ll} 
& -a_{t}-b_{t} X_{p}+c_{t} X_{n}+ \\
& -\left(c_{p}+d_{p} X_{p}+e_{p} X_{n}\right) \ln \left(1-b b_{p}\right) \\
& -\left(c_{n}+d_{n} X_{n}+e_{n} X_{p}\right) \ln \left(1+c b_{n}\right) \\
& -b g_{p}\left(X_{p}, X_{n}\right)-c g_{n}\left(X_{p}, X_{n}\right) \\
= & 0
\end{array}
$$

Equivalently we write

$$
\begin{aligned}
& -a_{t}-b_{t} X_{p}+c_{t} X_{n}+ \\
& -\left(c_{p}+d_{p} X_{p}+e_{p} X_{n}\right) \ln \left(1-b b_{p}\right) \\
& -\left(c_{n}+d_{n} X_{n}+e_{n} X_{p}\right) \ln \left(1+c b_{n}\right) \\
& +b\left(c_{p}+d_{p} X_{p}+e_{p} X_{n}\right) \ln \left(1-b_{p}\right) \\
& +c\left(c_{n}+d_{n} X_{n}+e_{n} X_{p}\right) \ln \left(1+b_{n}\right) \\
& =0
\end{aligned}
$$


Hence the required differential equations are

$$
\begin{aligned}
b_{t} & =-d_{p} \ln \left(1-b b_{p}\right)-e_{n} \ln \left(1+c b_{n}\right)+b d_{p} \ln \left(1-b_{p}\right)+c e_{n} \ln \left(1+b_{n}\right) \\
c_{t} & =d_{n} \ln \left(1+c b_{n}\right)+e_{p} \ln \left(1-b b_{p}\right)-b e_{p} \ln \left(1-b_{p}\right)-c d_{n} \ln \left(1+b_{n}\right) \\
a_{t} & =-c_{p} \ln \left(1-b b_{p}\right)-c_{n} \ln \left(1+c b_{n}\right)+b c_{p} \ln \left(1-b_{p}\right)+c c_{n} \ln \left(1+b_{n}\right)
\end{aligned}
$$

Let

$$
\begin{aligned}
y & =b b_{p} \\
w & =c b_{n}
\end{aligned}
$$

and write

$$
\begin{aligned}
y^{\prime} & =-b_{p} d_{p} \ln (1-y)-b_{p} e_{n} \ln (1+w)+y d_{p} \ln \left(1-b_{p}\right)+w \frac{b_{p} e_{n}}{b_{n}} \ln \left(1+b_{n}\right) \\
w^{\prime} & =b_{n} e_{p} \ln (1-y)+b_{n} d_{n} \ln (1+w)-y \frac{b_{n} e_{p}}{b_{p}} \ln \left(1-b_{p}\right)-w d_{n} \ln \left(1+b_{n}\right)
\end{aligned}
$$

The ordinary differential equations in $y, w$ are solved numerically for initial conditions

$$
\begin{aligned}
y(0, u) & =i u b_{p} \\
w(0, u) & =i u b_{n} .
\end{aligned}
$$

The solution for $a$ follows on numerical integration and hence we have the risk neutral characteristic function with the asymmetries of excitation and cross excitation.

\section{Scale, Speed, and Power Variation, Physically}

We report on estimates for the models for data on the top 50 underliers by option liquidity for 51 days taken 10 days apart. The ticker symbols for 50 underliers are presented in the Appendix. Two subsections present the results for the bilateral gamma model and the power variation model.

\subsection{Bilateral Gamma Results}

Figure 1 presents the quantiles for the scale parameter estimates for the upward and downward motion for the 2550 observations across names and days.

We observe that quite consistently the scale up is below the scale down. The mean difference is -480.3 basis points and the $t$ - statistic is -3.58 .

Figure 2 presents the quantiles for the speed parameter up and down in the case of the bilateral gamma model.

The speed up is consistently higher than the speed down. The mean difference is 1.5280 with a $t$ - statistic of 5.40. These bilateral gamma results are consistent with the observations in Madan and Wang (2017) and Madan, Schoutens and Wang (2017). 


\begin{tabular}{|c|c|c|}
\hline \multicolumn{3}{|c|}{ Bilateral Gamma Scale } \\
\hline \multicolumn{3}{|c|}{ Parameter Quantiles } \\
\hline Percentile & Scale up & Scale Down \\
\hline 1 & 8.0695 & 15.2352 \\
\hline 5 & 23.1531 & 29.9321 \\
\hline 10 & 30.6770 & 38.6222 \\
\hline 25 & 46.6507 & 56.9805 \\
\hline 50 & 69.2700 & 82.8834 \\
\hline 75 & 98.8349 & 108.5733 \\
\hline 90 & 144.8889 & 145.8174 \\
\hline 95 & 183.6948 & 181.5046 \\
\hline 99 & 281.1554 & 248.5961 \\
\hline
\end{tabular}

Figure 1: Percentiles for the scale parameters up and down in the bilateral gamma model estimated across 50 names on 51 days.

\begin{tabular}{|c|c|c|}
\hline \multicolumn{3}{|c|}{ Bilateral Gamma Speed } \\
Parameter Quantiles \\
\hline Percentile & Speed up & Speed Down \\
\hline 1 & 0.8164 & 0.5705 \\
\hline 5 & 1.0020 & 0.7533 \\
\hline 10 & 1.3981 & 1.0563 \\
\hline 25 & 1.7994 & 1.3983 \\
\hline 50 & 2.5716 & 2.0891 \\
\hline 75 & 5.6592 & 4.0452 \\
\hline 90 & 10.2364 & 7.6088 \\
\hline 95 & 106.1118 & 70.5060 \\
\hline 99 & 281.1554 & 248.5961 \\
\hline
\end{tabular}

Figure 2: Percentiles of speed parameters for the bilateral gamma model. 


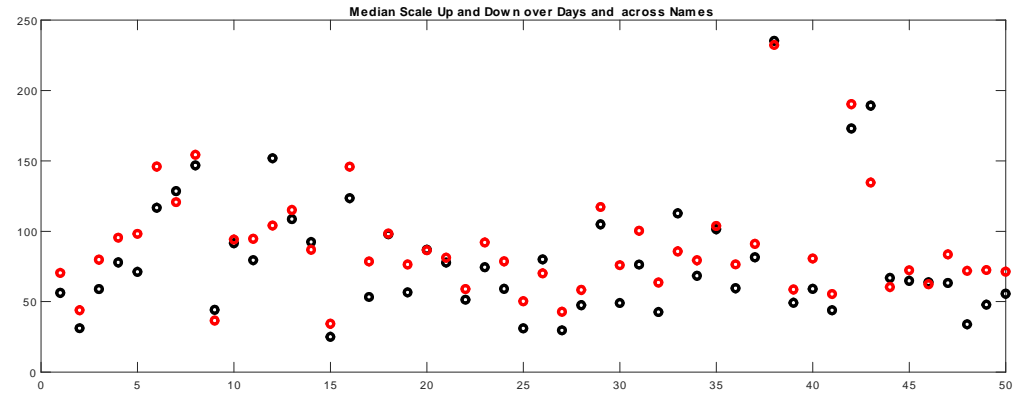

Figure 3: Median scale parameters up and down over days and across names. Blue circles are for the upward motion while red circles are for the downward motion.

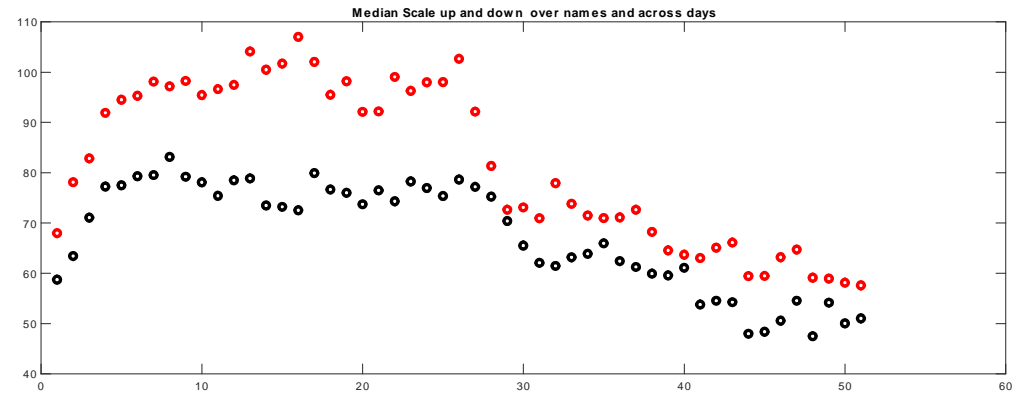

Figure 4: Median scale parameters up and down over names and across days. Blue circles are for the upward motion while red circles are for the downward motion.

Figure 3 presents the median scale parameters over days and across the 50 names. Generally the scale for the upward process is smaller tahn for the downward process.

Figure 4 presents the median scale parameters over names and across the 51 days. The median upward scale is uniformly lower than the median downward scale.

The corresponding Figures for the speed parameters are in Figures 5 and 6 The upward speed is higher than its downward counterpart.

\subsection{Power Variation Results}

The results for the scale and speed in the power variation model are similar to those for the bilateral gamma. The aggregate percentiles are presented in Figures 7 and 8 . 


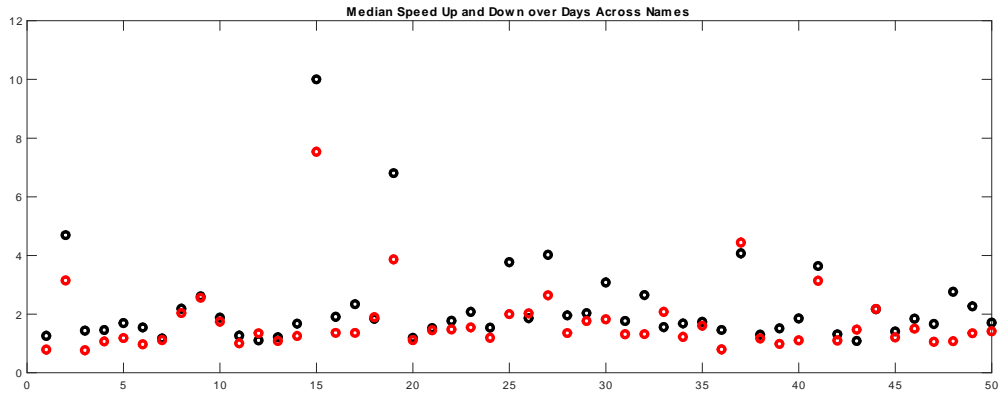

Figure 5: Median speed parameters up and down over days and across names. Blue circles are for the upward motion while red circles are for the downward motion.

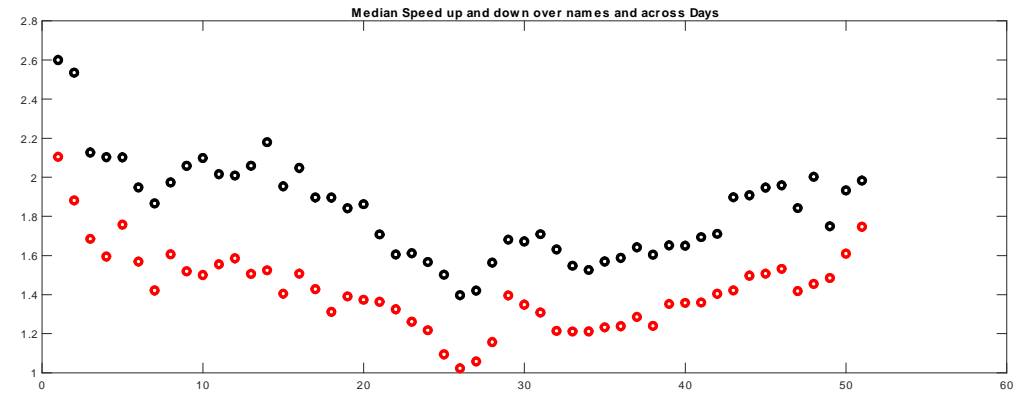

Figure 6: Median speed parameters up and down over days and across names. Blue circles are for the upward motion while red circles are for the downward motion. 


\begin{tabular}{|c|c|c|}
\hline \multicolumn{3}{|c|}{ Power Variation Scale } \\
\hline Parameter Quantiles \\
\hline Percentile & Scale up & Scale Down \\
\hline 1 & 6.0255 & 10.4475 \\
5 & 24.5602 & 29.4373 \\
10 & 30.8722 & 38.1046 \\
25 & 45.9535 & 59.1480 \\
50 & 66.5345 & 86.3381 \\
75 & 96.6792 & 115.9010 \\
90 & 140.5073 & 182.6168 \\
95 & 185.0352 & 254.1800 \\
99 & 282.0109 & 421.9139 \\
\hline
\end{tabular}

Figure 7: Percentiles for the scale parameters in the estimated power variation model.

\begin{tabular}{|c|c|c|}
\hline \multicolumn{3}{|c|}{ Power Variation Speed } \\
Parameter Quantiles \\
Percentile & Speed up & Speed down \\
\hline 1 & 0.0042 & 0.0025 \\
5 & 0.0685 & 0.0078 \\
10 & 0.8953 & 0.0306 \\
25 & 1.2052 & 0.8329 \\
50 & 1.5977 & 1.1744 \\
75 & 2.2732 & 1.7725 \\
90 & 3.6992 & 2.8109 \\
95 & 5.1864 & 4.3327 \\
\hline 99 & 12.7171 & 11.6011 \\
\hline
\end{tabular}

Figure 8: Percentiles for the speed parameters in the estimated power variation model. 


\begin{tabular}{|c|c|c|}
\hline \multicolumn{3}{|c|}{ Power Variation Parameter } \\
\hline \multicolumn{3}{|c|}{ Parameter Quantiles } \\
\hline Percentile & PV up & PV down \\
\hline 1 & 0.0000 & 0.0000 \\
5 & 0.0022 & 0.0009 \\
\hline 10 & 0.0061 & 0.0023 \\
\hline 25 & 0.0153 & 0.0080 \\
\hline 50 & 0.0273 & 0.0215 \\
\hline 75 & 0.0366 & 0.0365 \\
\hline 90 & 0.1112 & 0.6150 \\
\hline 95 & 0.4613 & 0.7876 \\
\hline 99 & 0.9405 & 0.9013 \\
\hline
\end{tabular}

Figure 9: Percentiles for the power variation parameters as estimated in the power variation model.

Additionally we have the levels for the power variation parameters. The percentiles for these parameters are presented in Figure 9.

At the lower percentiles the power variation parameter up is higher but this reverses at the higher percentiles. The mean is negative at -.0419 with a $t$-statistic of -8.55 . On this basis the power variation for the downward moves is higher and more powers have an infinite variation.

\section{Scale, Speed, and Power Variation, Risk Neu- trally}

Risk neutrally we first present the quartiles over the days for each of the 50 underliers for the Sato time scaling parameter, the bilateral gamma scale and speed parameters and the power variations as estimated in the power variation model. The estimations were conducted daily for a period of two years using the shorter maturities below a month. There are four Figures for time scaling 10, scale 11. speed 12 and power variation 13 The time scaling covers the bilateral gamma estimation and the power variation estimation. We observe similar results in the two estimations. The scale, speed and power variations quartiles are presented in six columns, three for the upward motion and three for the downward motion. We observe that the upward power variation is generally higher than the power variation for the downward process. 


\begin{tabular}{|c|c|c|c|c|c|c|}
\hline & \multicolumn{5}{|c|}{ Time Scaling Parameter Quartiles } & \\
\hline & \multicolumn{3}{|c|}{ Bilateral Gamma } & \multicolumn{3}{|c|}{ Power Variation } \\
\hline & Q1 & Q2 & Q3 & Q1 & Q2 & Q3 \\
\hline spy & 0.4909 & 0.5439 & 0.6097 & 0.4900 & 0.5445 & 0.6082 \\
\hline iwm & 0.4616 & 0.4996 & 0.5524 & 0.4574 & 0.4994 & 0.5544 \\
\hline qqq & 0.4797 & 0.5480 & 0.5897 & 0.4810 & 0.5489 & 0.5903 \\
\hline aapl & 0.4381 & 0.5087 & 0.6189 & 0.4380 & 0.5081 & 0.6204 \\
\hline $\mathrm{fb}$ & 0.4607 & 0.5436 & 0.6204 & 0.4604 & 0.5433 & 0.6220 \\
\hline nvda & 0.4268 & 0.4882 & 0.5859 & 0.4271 & 0.4940 & 0.5937 \\
\hline baba & 0.4270 & 0.4860 & 0.6043 & 0.4279 & 0.4869 & 0.6031 \\
\hline $\mathrm{mu}$ & 0.4582 & 0.5126 & 0.5839 & 0.4605 & 0.5180 & 0.5857 \\
\hline gld & 0.4242 & 0.4688 & 0.4934 & 0.4353 & 0.4957 & 1.1599 \\
\hline bac & 0.4275 & 0.4801 & 0.5375 & 0.4226 & 0.4773 & 0.5345 \\
\hline $\mathrm{msft}$ & 0.4321 & 0.4977 & 0.5817 & 0.4334 & 0.4948 & 0.5856 \\
\hline nflx & 0.4636 & 0.4984 & 0.5463 & 0.4635 & 0.4987 & 0.5464 \\
\hline C & 0.4230 & 0.4822 & 0.5332 & 0.4249 & 0.4802 & 0.5281 \\
\hline amzn & 0.4635 & 0.5510 & 0.6099 & 0.4635 & 0.5515 & 0.6105 \\
\hline eem & 0.4113 & 0.5016 & 0.5790 & 0.3832 & 0.4536 & 0.5245 \\
\hline tsla & 0.4488 & 0.5097 & 0.5969 & 0.4487 & 0.5096 & 0.5974 \\
\hline intc & 0.4159 & 0.4949 & 0.5631 & 0.4184 & 0.4945 & 0.5559 \\
\hline xop & 0.4560 & 0.4922 & 0.5506 & 0.4541 & 0.4913 & 0.5506 \\
\hline ewz & 0.4549 & 0.5049 & 0.5601 & 0.4508 & 0.5051 & 0.5579 \\
\hline jpm & 0.4529 & 0.4945 & 0.5599 & 0.4521 & 0.4965 & 0.5586 \\
\hline wfc & 0.4366 & 0.4862 & 0.5256 & 0.4349 & 0.4836 & 0.5246 \\
\hline dis & 0.4374 & 0.4946 & 0.5726 & 0.4375 & 0.4904 & 0.5845 \\
\hline gs & 0.4466 & 0.5020 & 0.5424 & 0.4466 & 0.5025 & 0.5426 \\
\hline xIf & 0.4130 & 0.4789 & 0.5389 & 0.4062 & 0.4823 & 0.5527 \\
\hline $\mathrm{t}$ & 0.4347 & 0.4979 & 0.5701 & 0.4336 & 0.4945 & 0.5600 \\
\hline xle & 0.4618 & 0.5027 & 0.5810 & 0.4605 & 0.5022 & 0.5833 \\
\hline rut & 0.5614 & 0.8102 & 1.4880 & 0.4860 & 0.6265 & 0.8334 \\
\hline wmt & 0.4343 & 0.4977 & 0.5459 & 0.4300 & 0.4969 & 0.5505 \\
\hline jd & 0.3923 & 0.4556 & 0.5341 & 0.3847 & 0.4499 & 0.5247 \\
\hline ba & 0.4567 & 0.4882 & 0.5706 & 0.4571 & 0.4891 & 0.5725 \\
\hline gm & 0.4345 & 0.4884 & 0.5702 & 0.4342 & 0.4912 & 0.5665 \\
\hline v & 0.4440 & 0.4992 & 0.5854 & 0.4318 & 0.5013 & 0.5870 \\
\hline bidu & 0.4361 & 0.4842 & 0.5367 & 0.4351 & 0.4845 & 0.5370 \\
\hline xom & 0.4698 & 0.5133 & 0.5683 & 0.4672 & 0.5155 & 0.5621 \\
\hline $\mathrm{cmg}$ & 0.4369 & 0.4940 & 0.5639 & 0.4368 & 0.4954 & 0.5695 \\
\hline $\mathrm{ndx}$ & 0.5168 & 0.5911 & 0.6323 & 0.5175 & 0.5871 & 0.6338 \\
\hline$g d x$ & 0.4365 & 0.4780 & 0.5460 & 0.4348 & 0.4736 & 0.5423 \\
\hline amd & 0.1979 & 0.3694 & 0.5601 & 0.1680 & 0.3588 & 0.5455 \\
\hline dia & 0.4921 & 0.5439 & 0.5986 & 0.4919 & 0.5464 & 0.5970 \\
\hline googl & 0.4546 & 0.5290 & 0.6030 & 0.4547 & 0.5287 & 0.6018 \\
\hline gild & 0.4071 & 0.4986 & 0.6202 & 0.4070 & 0.4990 & 0.6227 \\
\hline twtr & 0.4206 & 0.4831 & 0.5335 & 0.4206 & 0.4810 & 0.5371 \\
\hline wynn & 0.4560 & 0.5136 & 0.5485 & 0.4559 & 0.5152 & 0.5504 \\
\hline pypl & 0.3243 & 0.4028 & 0.5313 & 0.3148 & 0.4100 & 0.5299 \\
\hline ge & 0.4223 & 0.4920 & 0.5706 & 0.4189 & 0.4880 & 0.5668 \\
\hline Csco & 0.4742 & 0.5231 & 0.6053 & 0.4670 & 0.5160 & 0.6052 \\
\hline goog & 0.4549 & 0.5137 & 0.6077 & 0.4544 & 0.5136 & 0.6057 \\
\hline xlu & 0.4197 & 0.4723 & 0.5289 & 0.4165 & 0.4637 & 0.5233 \\
\hline hd & 0.4546 & 0.5262 & 0.6142 & 0.4548 & 0.5264 & 0.6123 \\
\hline cmcsa & 0.4536 & 0.4854 & 0.5828 & 0.4568 & 0.4847 & 0.5925 \\
\hline
\end{tabular}

Figure 10: Time scaling parameter quartiles as estimated for the bilateral gamma and power variation models. 


\begin{tabular}{|c|c|c|c|c|c|c|}
\hline & \multicolumn{5}{|c|}{ Bilateral Gamma Scale Parameters } & \\
\hline & \multirow[b]{2}{*}{ Q1 } & \multirow{2}{*}{$\begin{array}{c}\text { Scale up } \\
\text { Q2 }\end{array}$} & \multirow[b]{2}{*}{ Q3 } & \multicolumn{3}{|c|}{ Scale down } \\
\hline & & & & Q1 & Q2 & Q3 \\
\hline spy & 0.0017 & 0.0065 & 0.0288 & 0.1488 & 0.2011 & 0.2813 \\
\hline iwm & 0.0052 & 0.0146 & 0.0498 & 0.1489 & 0.1963 & 0.2526 \\
\hline qqq & 0.0028 & 0.0158 & 0.0493 & 0.1408 & 0.1975 & 0.2720 \\
\hline aapl & 0.0329 & 0.0507 & 0.1065 & 0.1627 & 0.2885 & 0.3561 \\
\hline $\mathrm{fb}$ & 0.0238 & 0.0554 & 0.0973 & 0.1633 & 0.2671 & 0.3597 \\
\hline nvda & 0.0488 & 0.1202 & 0.1826 & 0.1175 & 0.2077 & 0.5585 \\
\hline baba & 0.0691 & 0.0948 & 0.1489 & 0.1210 & 0.1925 & 0.2729 \\
\hline $\mathrm{mu}$ & 0.1668 & 0.2133 & 0.3047 & 0.2210 & 0.2851 & 0.4521 \\
\hline gld & 0.0001 & 0.0545 & 0.0800 & 0.0000 & 0.0322 & 0.0704 \\
\hline bac & 0.0740 & 0.1059 & 0.1247 & 0.1141 & 0.1429 & 0.1929 \\
\hline $\mathrm{msft}$ & 0.0303 & 0.0647 & 0.0929 & 0.1046 & 0.1594 & 0.2390 \\
\hline nflx & 0.0779 & 0.1112 & 0.1527 & 0.1249 & 0.1841 & 0.3481 \\
\hline C & 0.0638 & 0.0802 & 0.1077 & 0.1202 & 0.1453 & 0.2387 \\
\hline amzn & 0.0099 & 0.0468 & 0.0838 & 0.1399 & 0.2446 & 0.3564 \\
\hline eem & 0.0636 & 0.0823 & 0.1119 & 0.1494 & 0.1852 & 0.2408 \\
\hline tsla & 0.0791 & 0.0978 & 0.1517 & 0.2734 & 0.3572 & 0.4759 \\
\hline intc & 0.0587 & 0.0800 & 0.1079 & 0.0975 & 0.1515 & 0.1995 \\
\hline xop & 0.0963 & 0.1171 & 0.1346 & 0.1525 & 0.1808 & 0.2200 \\
\hline ewz & 0.0831 & 0.1203 & 0.1417 & 0.1802 & 0.2274 & 0.3191 \\
\hline jpm & 0.0190 & 0.0629 & 0.0753 & 0.1284 & 0.1592 & 0.2820 \\
\hline wfc & 0.0502 & 0.0691 & 0.0811 & 0.1047 & 0.1322 & 0.1840 \\
\hline dis & 0.0285 & 0.0689 & 0.0926 & 0.1115 & 0.1690 & 0.2863 \\
\hline gs & 0.0177 & 0.0322 & 0.0591 & 0.1846 & 0.2550 & 0.3509 \\
\hline xIf & 0.0547 & 0.0774 & 0.1039 & 0.1084 & 0.1371 & 0.1969 \\
\hline$t$ & 0.0256 & 0.0604 & 0.0760 & 0.1029 & 0.1494 & 0.3760 \\
\hline xle & 0.0535 & 0.0716 & 0.0841 & 0.1110 & 0.1380 & 0.1783 \\
\hline rut & 0.0090 & 0.0348 & 0.0955 & 0.2871 & 0.5400 & 5.2516 \\
\hline wmt & 0.0209 & 0.0596 & 0.0772 & 0.0960 & 0.1388 & 0.3078 \\
\hline jd & 0.1085 & 0.1670 & 0.2055 & 0.1591 & 0.2128 & 0.3194 \\
\hline ba & 0.0072 & 0.0174 & 0.0473 & 0.2261 & 0.3548 & 0.4986 \\
\hline $\mathrm{gm}$ & 0.0692 & 0.0936 & 0.1289 & 0.1265 & 0.1672 & 0.2591 \\
\hline $\mathrm{v}$ & 0.0097 & 0.0211 & 0.0718 & 0.1775 & 0.3184 & 0.4903 \\
\hline bidu & 0.0385 & 0.1052 & 0.1337 & 0.1381 & 0.2058 & 0.3141 \\
\hline xom & 0.0180 & 0.0412 & 0.0691 & 0.1048 & 0.1673 & 0.2236 \\
\hline $\mathrm{cmg}$ & 0.0764 & 0.1086 & 0.1862 & 0.1314 & 0.2244 & 0.4886 \\
\hline $\mathrm{ndx}$ & 0.0007 & 0.0010 & 0.0019 & 0.2017 & 0.2373 & 0.3174 \\
\hline $\mathrm{gdx}$ & 0.0879 & 0.1271 & 0.1792 & 0.1041 & 0.1500 & 0.2085 \\
\hline amd & 0.1714 & 0.2501 & 0.5041 & 0.1979 & 0.3536 & 0.6845 \\
\hline dia & 0.0011 & 0.0029 & 0.0216 & 0.1588 & 0.2480 & 0.3163 \\
\hline googl & 0.0139 & 0.0342 & 0.0632 & 0.1205 & 0.2140 & 0.3424 \\
\hline gild & 0.0716 & 0.1074 & 0.1632 & 0.1336 & 0.1905 & 0.3369 \\
\hline twtr & 0.2079 & 0.3186 & 0.6103 & 0.1406 & 0.2054 & 0.4232 \\
\hline wynn & 0.0881 & 0.1219 & 0.1685 & 0.1588 & 0.2131 & 0.3226 \\
\hline pypl & 0.0592 & 0.1024 & 0.1556 & 0.1370 & 0.2072 & 0.3654 \\
\hline ge & 0.0274 & 0.0613 & 0.0876 & 0.1049 & 0.1551 & 0.2880 \\
\hline Csco & 0.0576 & 0.0768 & 0.1055 & 0.1016 & 0.1394 & 0.2463 \\
\hline goog & 0.0170 & 0.0377 & 0.0712 & 0.1170 & 0.2034 & 0.3491 \\
\hline xlu & 0.0253 & 0.0449 & 0.0537 & 0.0716 & 0.0880 & 0.1330 \\
\hline hd & 0.0050 & 0.0161 & 0.0571 & 0.1623 & 0.2496 & 0.3360 \\
\hline cmcsa & 0.0189 & 0.0562 & 0.0878 & 0.1498 & 0.3018 & 0.7078 \\
\hline
\end{tabular}

Figure 11: Bilateral Gamma Scale Parameter Quartiles for the upward and downward process. 


\begin{tabular}{|c|c|c|c|c|c|c|}
\hline & \multirow[b]{3}{*}{ Q1 } & \multirow{3}{*}{$\begin{array}{c}\text { Speed Up } \\
\text { Q2 }\end{array}$} & \multicolumn{3}{|c|}{ Bilateral Gamma Speed } & \\
\hline & & & \multirow[b]{2}{*}{ Q3 } & \multicolumn{3}{|c|}{ Speed Down } \\
\hline & & & & Q1 & Q2 & Q3 \\
\hline spy & 1.1660 & 2.2727 & 7.1745 & 0.4268 & 1.0173 & 1.2310 \\
\hline iwm & 1.4599 & 1.8770 & 3.9507 & 1.1184 & 1.4611 & 1.8974 \\
\hline qqq & 1.1860 & 1.3058 & 1.5436 & 1.0687 & 1.2390 & 1.5079 \\
\hline aapl & 2.2648 & 7.5997 & 16.2361 & 0.2176 & 0.5145 & 2.1450 \\
\hline $\mathrm{fb}$ & 1.8907 & 2.9077 & 7.3533 & 0.8714 & 1.8255 & 2.4159 \\
\hline nvda & 2.0732 & 2.8918 & 9.9283 & 1.0022 & 2.3425 & 3.3220 \\
\hline baba & 2.4672 & 2.8869 & 5.9240 & 1.5934 & 2.6323 & 2.9270 \\
\hline $\mathrm{mu}$ & 1.3664 & 1.7400 & 2.1573 & 1.2263 & 1.6985 & 2.1082 \\
\hline gld & 1.3588 & 1.8983 & 2.1974 & 1.6293 & 1.9352 & 2.3642 \\
\hline bac & 1.6298 & 2.0364 & 2.3493 & 1.4785 & 2.0030 & 2.3277 \\
\hline $\mathrm{msft}$ & 1.6131 & 1.9174 & 2.5789 & 1.4326 & 1.8077 & 2.0271 \\
\hline $\mathrm{nflx}$ & 2.2676 & 2.9638 & 9.1310 & 0.8608 & 2.2676 & 2.9663 \\
\hline c & 1.6183 & 1.9113 & 2.2428 & 1.6012 & 1.8761 & 2.1479 \\
\hline amzn & 3.3951 & 7.0184 & 16.4788 & 0.3797 & 2.3287 & 4.1081 \\
\hline eem & 0.7638 & 1.4086 & 1.5996 & 0.6065 & 1.1842 & 1.5377 \\
\hline tsla & 3.2779 & 6.0747 & 12.2221 & 0.5022 & 0.8655 & 2.2228 \\
\hline intc & 1.3363 & 1.6692 & 2.3964 & 1.2656 & 1.6050 & 2.3131 \\
\hline xop & 2.0181 & 2.3942 & 3.0960 & 2.0181 & 2.3942 & 3.0960 \\
\hline ewz & 1.8418 & 2.2983 & 2.8260 & 1.7648 & 2.0732 & 2.6004 \\
\hline jpm & 1.4878 & 1.8145 & 2.2953 & 0.9914 & 1.6269 & 1.9803 \\
\hline wfc & 1.6093 & 2.0671 & 2.5764 & 1.4635 & 1.8702 & 2.2930 \\
\hline dis & 1.5347 & 1.8210 & 2.5774 & 0.3916 & 1.5133 & 1.7813 \\
\hline gs & 1.9904 & 12.4919 & 24.9679 & 0.3844 & 0.6878 & 1.7733 \\
\hline xlf & 0.7495 & 1.1514 & 1.4458 & 0.6660 & 1.1317 & 1.3737 \\
\hline $\mathrm{t}$ & 1.2687 & 1.9073 & 10.4550 & 0.3377 & 1.1865 & 1.6894 \\
\hline xle & 1.7543 & 2.0486 & 2.7159 & 1.6998 & 1.9934 & 2.6629 \\
\hline rut & 1.2139 & 2.0814 & 2.9077 & 0.7672 & 1.4032 & 2.3554 \\
\hline wmt & 1.7819 & 2.0735 & 2.5287 & 1.6036 & 1.8595 & 2.2759 \\
\hline $\mathrm{jd}$ & 1.1635 & 1.8478 & 2.5828 & 1.0753 & 1.8063 & 2.4040 \\
\hline ba & 1.8458 & 2.2346 & 20.9592 & 0.4044 & 1.6215 & 1.9286 \\
\hline $\mathrm{gm}$ & 1.4046 & 1.8246 & 2.2373 & 1.3356 & 1.6553 & 2.0688 \\
\hline$v$ & 1.3530 & 1.6548 & 2.1458 & 0.9334 & 1.3783 & 1.6730 \\
\hline bidu & 2.0034 & 2.5559 & 4.2235 & 1.4217 & 2.2390 & 2.7948 \\
\hline xom & 1.5901 & 2.0127 & 4.4866 & 1.2429 & 1.6518 & 2.0782 \\
\hline $\mathrm{cmg}$ & 1.8774 & 2.7128 & 5.1931 & 0.8100 & 2.0914 & 2.6684 \\
\hline $\mathrm{ndx}$ & 0.5885 & 1.5433 & 15.0897 & 0.7174 & 1.2880 & 1.9268 \\
\hline$g d x$ & 2.7627 & 3.1249 & 3.6969 & 2.7626 & 3.1249 & 3.6969 \\
\hline amd & 1.0208 & 1.5190 & 1.8392 & 0.7965 & 1.5189 & 1.8392 \\
\hline dia & 1.1058 & 1.3158 & 2.6515 & 0.9729 & 1.1279 & 1.4110 \\
\hline googl & 2.0420 & 3.2984 & 6.9212 & 0.9422 & 2.1821 & 3.4433 \\
\hline gild & 1.4755 & 1.7794 & 2.3373 & 0.8481 & 1.5186 & 1.8138 \\
\hline twtr & 0.4521 & 1.4651 & 2.1824 & 1.4460 & 2.1793 & 4.4879 \\
\hline wynn & 2.3121 & 2.9177 & 4.6339 & 1.8009 & 2.4848 & 3.8277 \\
\hline pypl & 1.0229 & 1.5737 & 2.8127 & 0.5957 & 1.1233 & 1.8150 \\
\hline ge & 1.3315 & 1.6996 & 2.3770 & 0.7596 & 1.3603 & 1.7693 \\
\hline csco & 1.2855 & 1.5975 & 2.3798 & 1.1466 & 1.5123 & 2.2079 \\
\hline goog & 2.1679 & 3.7828 & 15.2800 & 0.5975 & 1.6820 & 2.8302 \\
\hline xlu & 1.5067 & 1.8145 & 2.5142 & 1.4027 & 1.7102 & 2.3535 \\
\hline hd & 1.8468 & 2.9383 & 7.1605 & 0.5714 & 1.7242 & 2.2165 \\
\hline cmcsa & 1.2818 & 1.5909 & 7.1423 & 0.1924 & 1.1917 & 1.4963 \\
\hline
\end{tabular}

Figure 12: Bilateral gamma quartiles for the speed parameter of the upward and downward motion. 


\begin{tabular}{|c|c|c|c|c|c|c|}
\hline & & \multicolumn{4}{|c|}{ Power Variation } & \\
\hline & & PV up & & & PV down & \\
\hline & Q1 & Q2 & Q3 & Q1 & Q2 & Q3 \\
\hline spy & 0.3906 & 0.9652 & 1.6922 & 0.1384 & 0.2701 & 1.0182 \\
\hline iwm & 0.2530 & 0.3246 & 1.6985 & 0.1090 & 0.1805 & 0.2221 \\
\hline qqq & 0.2693 & 0.4107 & 1.6501 & 0.1443 & 0.2002 & 0.2379 \\
\hline aapl & 0.2437 & 1.0729 & 1.2716 & 0.0674 & 0.1266 & 0.1871 \\
\hline $\mathrm{fb}$ & 0.2422 & 0.3246 & 1.4393 & 0.1248 & 0.1533 & 0.2052 \\
\hline nvda & 0.2110 & 0.2976 & 0.9281 & 0.1317 & 0.1781 & 0.2446 \\
\hline baba & 0.1742 & 0.2382 & 0.3495 & 0.1149 & 0.1502 & 0.1995 \\
\hline $\mathrm{mu}$ & 0.2022 & 0.2483 & 0.3551 & 0.1641 & 0.2001 & 0.2473 \\
\hline gld & 0.0901 & 0.1576 & 0.2222 & 0.1302 & 0.1868 & 0.4061 \\
\hline bac & 0.1276 & 0.1684 & 0.2015 & 0.1406 & 0.1794 & 0.2084 \\
\hline msft & 0.1463 & 0.1943 & 0.3980 & 0.1152 & 0.1741 & 0.2073 \\
\hline$n f l x$ & 0.1740 & 0.3148 & 0.7289 & 0.1366 & 0.1910 & 0.3065 \\
\hline c & 0.1645 & 0.1976 & 0.2655 & 0.1155 & 0.1497 & 0.1942 \\
\hline amzn & 0.3252 & 0.8468 & 1.7576 & 0.1491 & 0.2559 & 0.6570 \\
\hline eem & 0.1726 & 0.2511 & 0.4582 & 0.1695 & 0.1948 & 0.4094 \\
\hline tsla & 0.4088 & 0.6704 & 0.9637 & 0.1595 & 0.2189 & 0.2905 \\
\hline intc & 0.1488 & 0.1907 & 0.2465 & 0.1278 & 0.1800 & 0.2065 \\
\hline xop & 0.1183 & 0.1610 & 0.2029 & 0.1387 & 0.1615 & 0.1828 \\
\hline ewz & 0.1451 & 0.2005 & 0.2595 & 0.1507 & 0.1818 & 0.2046 \\
\hline jpm & 0.1740 & 0.2605 & 1.3566 & 0.0863 & 0.1449 & 0.1765 \\
\hline wfc & 0.1353 & 0.1674 & 0.2569 & 0.1096 & 0.1552 & 0.1900 \\
\hline dis & 0.2133 & 0.2775 & 0.9464 & 0.1018 & 0.1548 & 0.2303 \\
\hline gs & 0.3895 & 1.3240 & 1.5566 & 0.1058 & 0.1577 & 0.2432 \\
\hline xIf & 0.1868 & 0.2493 & 0.3897 & 0.1337 & 0.1943 & 0.2620 \\
\hline $\mathrm{t}$ & 0.1588 & 0.2334 & 0.3798 & 0.0791 & 0.1356 & 0.2249 \\
\hline xle & 0.1290 & 0.1693 & 0.2084 & 0.1282 & 0.1482 & 0.1791 \\
\hline rut & 0.2901 & 0.8035 & 1.2458 & 0.2223 & 0.4443 & 1.5857 \\
\hline wmt & 0.1371 & 0.1848 & 0.2905 & 0.0955 & 0.1674 & 0.2037 \\
\hline $\mathrm{jd}$ & 0.1572 & 0.2445 & 0.3467 & 0.1308 & 0.1830 & 0.3225 \\
\hline ba & 0.3838 & 1.6012 & 1.9198 & 0.0543 & 0.0936 & 0.1994 \\
\hline $\mathrm{gm}$ & 0.1608 & 0.2202 & 0.3037 & 0.1203 & 0.1609 & 0.2141 \\
\hline $\mathrm{v}$ & 0.2673 & 0.4021 & 1.7686 & 0.0701 & 0.1430 & 0.2169 \\
\hline bidu & 0.2373 & 0.3172 & 1.0920 & 0.1317 & 0.1744 & 0.2532 \\
\hline xom & 0.1331 & 0.2469 & 0.3464 & 0.0853 & 0.1448 & 0.1820 \\
\hline $\mathrm{cmg}$ & 0.2797 & 0.4261 & 0.8315 & 0.1719 & 0.2877 & 0.4915 \\
\hline$n d x$ & 0.3616 & 0.9888 & 1.8956 & 0.5307 & 1.2168 & 1.4136 \\
\hline $\mathrm{gdx}$ & 0.0931 & 0.1358 & 0.1650 & 0.0956 & 0.1282 & 0.1699 \\
\hline amd & 0.2258 & 0.3337 & 0.6271 & 0.1757 & 0.3324 & 0.5078 \\
\hline dia & 0.3195 & 1.1030 & 1.9890 & 0.1338 & 0.1953 & 0.2865 \\
\hline googl & 0.2780 & 0.4416 & 1.3564 & 0.1666 & 0.2254 & 0.3148 \\
\hline gild & 0.1927 & 0.2512 & 0.3446 & 0.1370 & 0.1789 & 0.2287 \\
\hline twtr & 0.1413 & 0.2249 & 0.3287 & 0.2011 & 0.2787 & 0.5796 \\
\hline wynn & 0.2066 & 0.2755 & 0.6147 & 0.1042 & 0.1521 & 0.2001 \\
\hline pypl & 0.1913 & 0.2859 & 0.3817 & 0.1231 & 0.2120 & 0.2941 \\
\hline ge & 0.1660 & 0.2330 & 0.3445 & 0.1255 & 0.1816 & 0.2482 \\
\hline Csco & 0.1421 & 0.2138 & 0.3094 & 0.1169 & 0.1872 & 0.2660 \\
\hline goog & 0.3022 & 0.5580 & 1.5669 & 0.1384 & 0.2142 & 0.3009 \\
\hline xlu & 0.0974 & 0.1534 & 0.1984 & 0.0701 & 0.1233 & 0.1807 \\
\hline hd & 0.2701 & 1.1720 & 1.9824 & 0.0622 & 0.1414 & 0.2090 \\
\hline cmcsa & 0.2460 & 0.3744 & 1.5107 & 0.0885 & 0.1490 & 0.2449 \\
\hline
\end{tabular}

Figure 13: Quartiles for the power variation parameters for the upward and downward motion. 


\section{Equilibrium Return Distributions}

Return distributions in any parametric class may be estimated daily on the basis of immediately past return data. The result is a time series of parameters that characterize the distribution of price motion. In principle each parameter has limits set by where the other parameters are. In the simplest terms with just two parameters of mean and variance we understand that the mean is risk compensation for the risk represented by the variance. But equally given the mean the variance can get too small relative to this mean for otherwise the retsun would become too much of a good deal. Hence one may employ the time series of parameters to estimate how each parameter is potentially responding to the other. The relationships between parameters may however be quite nonlinear as they are entities of different scales. With a view to matching the scales one may consider the $n^{t h}$ root of the $n^{\text {th }}$ moment. However, such a procedure requires access to densities and their moments that can get quite complicated. Furthermore, one is aggregating across all sizes and not separating the upward and downward motions. We work instead with the $n^{\text {th }}$ root of the $n^{\text {th }}$ power variation taken separately for the upward and downward motions. For the bilateral gamma model the first two variations on each side suffice. For power variation with finite variation on each side the first second and thrid variations on each side suffice. In the case of infinite variation we use the second, third and fourth variations on each side. In each case we first determine a one to one mapping from the parameters to the variations being employed and back.

We denote the variations being employed as

$$
\begin{aligned}
& m_{n}^{p}=\left(\int_{0}^{\infty} x^{n} k(x) d x\right)^{\frac{1}{n}} \\
& m_{n}^{n}=\left(\int_{0}^{\infty} x^{n} k(-x) d x\right)^{\frac{1}{n}}
\end{aligned}
$$

For the bilateral gamma we have

$$
\begin{aligned}
m_{1}^{p} & =b_{p} c_{p} \\
m_{2}^{p} & =b_{p} \sqrt{c_{p}} \\
m_{1}^{n} & =b_{n} c_{n} \\
m_{2}^{n} & =b_{n} \sqrt{c_{n}}
\end{aligned}
$$


and the reverse mapping is

$$
\begin{aligned}
c_{p} & =\left(\frac{m_{1}^{p}}{m_{2}^{p}}\right)^{2} \\
b_{p} & =\frac{\left(m_{2}^{p}\right)^{2}}{m_{1}^{p}} \\
c_{n} & =\left(\frac{m_{1}^{n}}{m_{2}^{n}}\right)^{2} \\
b_{n} & =\frac{\left(m_{2}^{n}\right)^{2}}{m_{1}^{n}} .
\end{aligned}
$$

For power variation in the finite variation case we have on the positive side, with similar expressions for the negative side, and suppressing the subscript $p$ on the $b, c, y$ parameters and the superscript $p$ on the variations, that

$$
\begin{aligned}
& m_{1}=b^{1-y}(c \Gamma(1-y)) \\
& m_{2}=b^{1-y / 2}(c \Gamma(2-y))^{\frac{1}{2}} \\
& m_{3}=b^{1-y / 3}(c \Gamma(3-y))^{\frac{1}{3}}
\end{aligned}
$$

It follows that

$$
\begin{aligned}
\log \left(\frac{m_{3}^{3} m_{1}}{m_{2}^{4}}\right) & =\ln \Gamma(3-y)+\ln \Gamma(1-y)-2 \ln \Gamma(2-y) \\
b & =\frac{m_{2}^{2}}{m_{1}} \frac{\Gamma(1-y)}{\Gamma(2-y)} \\
c & =\frac{m_{1}}{b^{1-y} \Gamma(1-y)} .
\end{aligned}
$$

For power variation and infinite variation we have

$$
\begin{aligned}
& m_{2}=b^{1-y / 2}(c \Gamma(2-y))^{\frac{1}{2}} \\
& m_{3}=b^{1-y / 3}(c \Gamma(3-y))^{\frac{1}{3}} \\
& m_{4}=b^{1-y / 4}(c \Gamma(4-y))^{\frac{1}{4}}
\end{aligned}
$$

and for the reverse

So

$$
\begin{aligned}
\log \left(\frac{m_{4}^{4} m_{2}^{2}}{m_{3}^{6}}\right) & =\log (\Gamma(4-y))+\log (\Gamma(2-y)-2 \log (\Gamma(3-y)) \\
b & =\frac{\Gamma(2-y)}{\Gamma(3-y)} \frac{m_{3}^{3}}{m_{2}^{2}} \\
c & =\frac{m_{2}^{2}}{b^{2-y} \Gamma(2-y)} .
\end{aligned}
$$


Given a time series of parameters we may employ linear regression to estimate each parameter as a linear function of the others with a constant term estimated by the vector $\kappa$ for all the variations used. One may then seek a fixed point of these response functions for the variations $v$. The result is an equation system of the form

$$
(I-B) v=\kappa
$$

with fixed point solution for the variations of

$$
v=(1-B)^{-1} \kappa .
$$

The equilibrium variations may then be transformed into equilibrium parameters using the inverse mapptings outlined. This generally works well but on occasion the matrix being inverted may be ill conditioned with solutions producing outliers that may be deleted or interpolated from the other good points.

With a view towards bypassing these inversion issues we replaced the linear regression by a nonlinear support vector machine regression based on a Gaussian kernel. These nonlinear predictions are generally superior to linear regression and more importantly map into a compact range that is appropriate for variations derived from parameters of motion. The result is a nonlinear response mapping of the form

$$
v^{\prime}=F(v)
$$

and a fixed point is easily as

$$
v^{*}=\lim _{n \rightarrow \infty} F^{n}\left(v_{0}\right)
$$

where $F^{n}$ is an n-fold composition of $F$ with itself. For a starting value we used the median point in the data set employed in estimating $F$. We employed the past 126 days or six months of data on parameter values and variations to estimate the support vector machine regressions delivering the response function $F$. Two subsections present the results on equilibrium return distributions using bilateral gamma parameters, power variation parameters with finite variation imposed. Infinite variation was not observed in the physical process and this case is taken up risk neutrally.

\subsection{Bilateral Gamma Equilibrium Distributions}

Figure 14 presents a graph of the four bilateral gamma parameters as they were estimated from the time series data on the ticker $S P Y$ along with the equilibrium levels based on three months of support vector machine regressions and six months of linear regressions.

We also present in four Figures 15, 16, 17 and 18 with four parameter values and the two associated equilibrium values at intervals of a month or 21 days. 

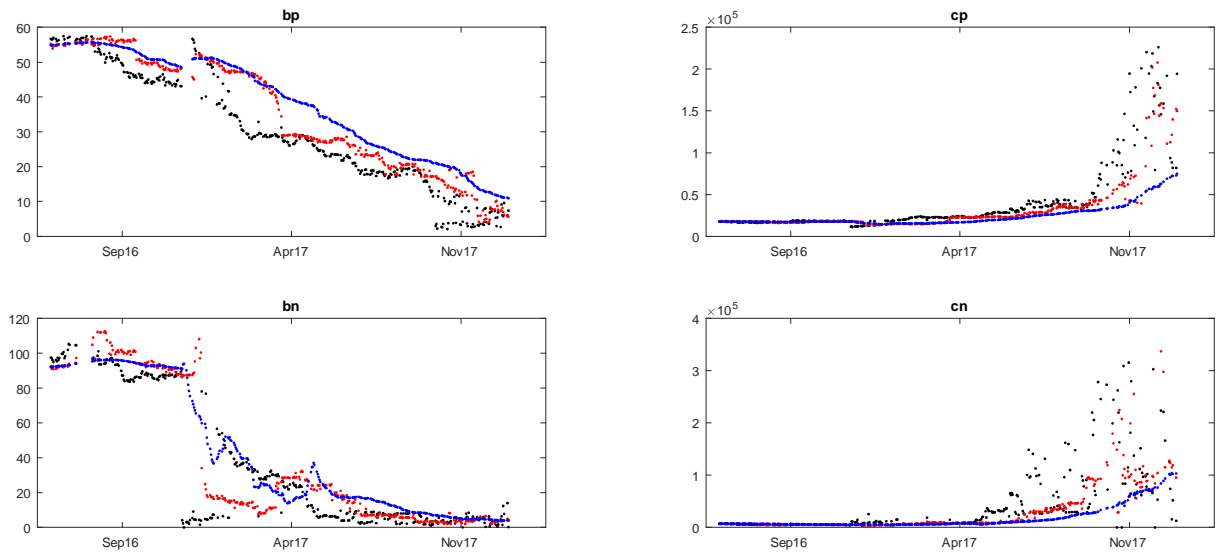

Figure 14: Bilateral Gamma parameter Values in Black and the support vector machine regression equilibrium values in red. Also presented are equilibrium values based on linear regression in blue.

\subsection{Power Variation under Finite Variation}

For power variation equilibrium distributions of finite variation results presented are for the ticker $M S F T$. Figure 19 presents graphs of the six parameters and the two equilibrium constructions.

Additionally we present Figures 20 and 21 providing the estimated and equilibrium values for the finite variation time scaling coefficients.

\section{Equilibrium Volatility Surfaces}

Risk neutrally one works with compensated jump martingales adjusted by an external drift to fit the forward price. Here we allow for infinite variation and present in this section the equilibrium volatility surfaces for power variation in the infinite variation context. The option pricing model is extended away from a Lévy process to that of a time scaled self decomposable law with an additional scaling parameter. In developing the equilibrium levels we added this scaling parameter into the linear and support vector machine parametric response regressions before solving for the required fixed points.

The results presented are for the ticker $A M Z N$. Figure 22 presents the graphs for the data and the equilibrium on the six power variation parameters. Figure 23 present the data and equilibrium for the time scaling parameter.

We observe that the equilibrium construction smooths out many extremal observations. The equilibrium estimation of the power variation parameters is somewhat noisy but here we used an interpolation procedure for the estimation 


\begin{tabular}{|c|c|c|c|}
\hline \multicolumn{2}{|c|}{ Scale Up in Basis Points } & \\
dates & data & rsvm & linear \\
\hline 20160705 & 56.6688 & 55.2337 & 54.8597 \\
\hline 20160803 & 56.0323 & 55.2900 & 55.4632 \\
\hline 20160901 & 52.8721 & 56.6588 & 55.4128 \\
\hline 20161003 & 47.0783 & 55.9848 & 54.0870 \\
\hline 20161101 & 43.7929 & 49.3531 & 50.7198 \\
\hline 20161201 & 44.6093 & 47.9262 & 48.9162 \\
\hline 20170103 & 51.0365 & 51.2783 & 50.9231 \\
\hline 20170202 & 34.9028 & 47.0671 & 49.0542 \\
\hline 20170306 & 29.1535 & 46.0736 & 44.4320 \\
\hline 20170404 & 28.3679 & 28.9968 & 40.4409 \\
\hline 20170504 & 27.4871 & 28.7808 & 37.7133 \\
\hline 20170605 & 23.4722 & 28.1506 & 32.7626 \\
\hline 20170705 & 17.9729 & 25.3980 & 28.4060 \\
\hline 20170803 & 17.3575 & 20.5326 & 25.3334 \\
\hline 20170901 & 19.4758 & 20.6744 & 22.2434 \\
\hline 20171003 & 3.5550 & 17.2317 & 20.9757 \\
\hline 20171101 & 3.3070 & 17.6325 & 18.0325 \\
\hline 20171201 & 4.0786 & 5.0293 & 12.7998 \\
\hline
\end{tabular}

Figure 15: Scale up in basis points at monthly intervals along with support vector machine regression and linear regression equilibrium values based on three and six months of past parameter estimates. 


\begin{tabular}{|c|c|c|c|}
\hline & Speed Up & & \\
\hline dates & data & rsvm & linear \\
\hline 20160705 & 1.7900 & 1.7721 & 1.7638 \\
\hline 20160803 & 1.7335 & 1.7826 & 1.7665 \\
\hline 20160901 & 1.6545 & 1.6669 & 1.7483 \\
\hline 20161003 & 1.8656 & 1.6572 & 1.7303 \\
\hline 20161101 & 1.8466 & 1.7915 & 1.7562 \\
\hline 20161201 & 1.7846 & 1.7630 & 1.7677 \\
\hline 20170103 & 1.3199 & 1.4234 & 1.5584 \\
\hline 20170202 & 1.9628 & 1.4933 & 1.5608 \\
\hline 20170306 & 2.2682 & 1.4893 & 1.5598 \\
\hline 20170404 & 2.2725 & 2.2388 & 1.6641 \\
\hline 20170504 & 2.3368 & 2.2565 & 1.7870 \\
\hline 20170605 & 2.8311 & 2.2802 & 2.0210 \\
\hline 20170705 & 4.2562 & 2.5502 & 2.3247 \\
\hline 20170803 & 4.1801 & 3.3457 & 2.6174 \\
\hline 20170901 & 3.3915 & 3.4093 & 3.0160 \\
\hline 20171003 & 65.3316 & 4.3146 & 3.2503 \\
\hline 20171101 & 19.4531 & 4.2712 & 4.0449 \\
\hline 20171201 & 7.9364 & 16.8505 & 5.9183 \\
\hline
\end{tabular}

Figure 16: Speed up at monthly intervals along with support vector machine regression and linear regression equilibrium values based on three and six months of past parameter estimates. 


\begin{tabular}{|c|c|c|c|}
\hline \multicolumn{2}{|c|}{ Scale Down in Basis Points } & \\
\hline dates & data & rsvm & linear \\
\hline 20160705 & 97.5166 & 92.4537 & 92.1008 \\
\hline 20160803 & 104.5373 & 94.5953 & 93.9652 \\
\hline 20160901 & 93.7104 & 111.9504 & 96.0882 \\
\hline 20161003 & 84.4108 & 99.7066 & 95.3995 \\
\hline 20161101 & 87.5904 & 95.6318 & 92.6894 \\
\hline 20161201 & 87.7581 & 87.7544 & 91.4929 \\
\hline 20170103 & 4.8382 & 24.8247 & 59.4491 \\
\hline 20170202 & 42.4881 & 15.2961 & 50.0202 \\
\hline 20170306 & 31.9689 & 12.0869 & 23.1589 \\
\hline 20170404 & 26.6133 & 28.5216 & 19.4829 \\
\hline 20170504 & 12.0212 & 26.9024 & 23.9987 \\
\hline 20170605 & 6.4355 & 19.8677 & 18.6010 \\
\hline 20170705 & 2.1819 & 13.6362 & 17.2025 \\
\hline 20170803 & 4.9405 & 7.2429 & 14.4124 \\
\hline 20170901 & 8.1844 & 5.6022 & 9.1565 \\
\hline 20171003 & 3.0685 & 3.1646 & 6.9629 \\
\hline 20171101 & 5.2836 & 3.1877 & 4.9735 \\
\hline 20171201 & 1705.8958 & 5.7175 & 4.5978 \\
\hline
\end{tabular}

Figure 17: Scale down up in basis points at monthly intervals along with support vector machine regression and linear regression equilibrium values based on three and six months of past parameter estimates. 


\begin{tabular}{|c|c|c|c|}
\hline \multicolumn{3}{|c|}{ Speed Down } & \\
\hline dates & data & rsvm & linear \\
\hline 20160705 & 0.7000 & 0.7090 & 0.7051 \\
\hline 20160803 & 0.5964 & 0.6931 & 0.6975 \\
\hline 20160901 & 0.5018 & 0.5156 & 0.6570 \\
\hline 20161003 & 0.5876 & 0.5296 & 0.6034 \\
\hline 20161101 & 0.5203 & 0.5041 & 0.5584 \\
\hline 20161201 & 0.4676 & 0.5217 & 0.5336 \\
\hline 20170103 & 0.9014 & 0.3256 & 0.5244 \\
\hline 20170202 & 0.5395 & 0.5617 & 0.5296 \\
\hline 20170306 & 0.6689 & 0.7688 & 0.5990 \\
\hline 20170404 & 0.7522 & 0.7223 & 0.6772 \\
\hline 20170504 & 1.4506 & 0.7633 & 0.6848 \\
\hline 20170605 & 3.1123 & 0.9646 & 0.9192 \\
\hline 20170705 & 14.8612 & 1.4015 & 1.1367 \\
\hline 20170803 & 6.1002 & 3.3534 & 1.4765 \\
\hline 20170901 & 2.7761 & 4.6725 & 2.4932 \\
\hline 20171003 & 63.6707 & 9.6594 & 3.4505 \\
\hline 20171101 & 6.0913 & 9.7170 & 5.9497 \\
\hline 20171201 & 0.0000 & 8.4147 & 7.6773 \\
\hline
\end{tabular}

Figure 18: Speed down up points at monthly intervals along with support vector machine regression and linear regression equilibrium values based on three and six months of past parameter estimates. 

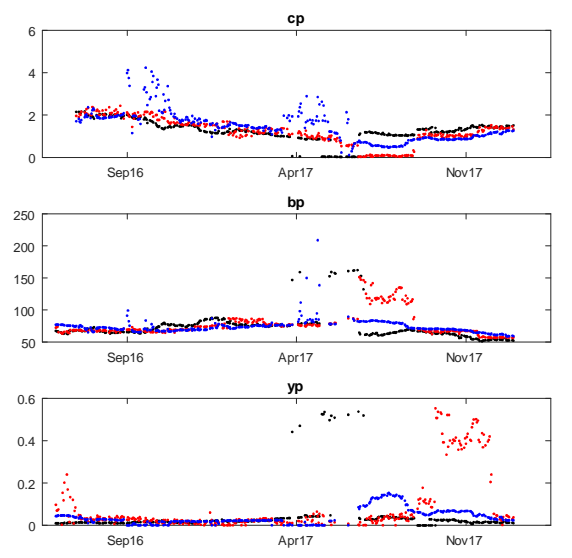

Figure 19: Power Variation parameter Values in Black and the support vector machine regression finite variation equilibrium values in red. Also presented are equilibrium values based on linear regression in blue.

of this parameter as opposed to a proper equation solve. We anticipate better results with a proper equation solve.

\section{Asset Pricing Implications}

Classically asset pricing models seek to explain differences in expected returns in terms of return covariations with systematic factors representing undiversifiable risks. Alternatively, the absence of arbitrage links to martingale conditions under a measure change and it is the covariation with the change of measure density that is critical in explaining expected returns. The two may be linked by expressing the return on the change of measure density as being driven by candidate systematic factor selections in constructing multifactor arbitrage pricing models. Both the equilibrium and arbitrage pricing approaches to asset pricing take an economy wide perspective seeking portfolios that maximize expected utilities or seek arbitrage opportunities. The asset pricing theory is an implication of economy wide perspectives defining efficient portfolios or excluding arbitrages. Explaining the cross section of expected returns has been a classical objective in finance theory.

The focus of attention in this section is somewhat different. We seek to explain, not expected returns, but the full equilibrium return distribution. The equilibrium return distribution construction recognizes that each aspect of the return distribution is continuously responding to and reacting to the other aspects. The question then is to ask what equilibrium return distributions can prevail for asset returns taken one asset at a time for a fixed and small return horizon. Generally, covariations and correlations occur over the longer time 


\begin{tabular}{|c|c|c|c|}
\hline \multicolumn{2}{|c|}{ Power Variation Up } & \\
\hline dates & data & rsvm & linear \\
\hline 20160705 & 0.0105 & 0.0310 & 0.0440 \\
\hline 20160803 & 0.0153 & 0.0230 & 0.0420 \\
\hline 20160901 & 0.0118 & 0.0240 & 0.0290 \\
\hline 20161003 & 0.0139 & 0.0610 & 0.0010 \\
\hline 20161101 & 0.0181 & 0.0100 & 0.0010 \\
\hline 20161201 & 0.0160 & 0.0300 & 0.0200 \\
\hline 20170103 & 0.0198 & 0.0140 & 0.0160 \\
\hline 20170202 & 0.0179 & 0.0250 & 0.0220 \\
\hline 20170306 & 0.0235 & 0.0210 & 0.0240 \\
\hline 20170404 & 0.0342 & 0.0020 & 0.0010 \\
\hline 20170504 & 0.0426 & 0.0350 & 0.0010 \\
\hline 20170605 & 0.5563 & 0.0620 & 0.0480 \\
\hline 20170705 & 0.0266 & 0.5270 & 0.0900 \\
\hline 20170803 & 0.0370 & 0.3860 & 0.1490 \\
\hline 20170901 & 0.0342 & 0.2400 & 0.0630 \\
\hline 20171003 & 0.0254 & 0.0410 & 0.0640 \\
\hline 20171101 & 0.0191 & 0.0300 & 0.0680 \\
\hline 20171201 & 0.0161 & 0.0010 & 0.0360 \\
\hline
\end{tabular}

Figure 20: Power Variation up at monthly intervals along with support vector machine regression and linear regression equilibrium values based on three and six months of past parameter estimates. 


\begin{tabular}{|c|c|c|c|}
\hline \multicolumn{3}{|c|}{ Power Variation Down } & \\
\hline dates & data & rsvm & linear \\
\hline 20160705 & 0.0020 & 0.0160 & 0.0240 \\
\hline 20160803 & 0.0049 & 0.0210 & 0.0200 \\
\hline 20160901 & 0.0029 & 0.0030 & 0.0180 \\
\hline 20161003 & 0.0058 & 0.0110 & 0.0010 \\
\hline 20161101 & 0.0095 & 0.0010 & 0.0010 \\
\hline 20161201 & 0.0086 & 0.0010 & 0.0110 \\
\hline 20170103 & 0.0124 & 0.0130 & 0.0070 \\
\hline 20170202 & 0.0124 & 0.0130 & 0.0110 \\
\hline 20170306 & 0.0134 & 0.0450 & 0.0150 \\
\hline 20170404 & 0.0202 & 0.0150 & 0.0010 \\
\hline 20170504 & 0.0266 & 0.0600 & 0.0010 \\
\hline 20170605 & 0.4593 & 0.1310 & 0.0350 \\
\hline 20170705 & 0.0156 & 0.2940 & 0.0590 \\
\hline 20170803 & 0.0171 & 0.2680 & 0.1030 \\
\hline 20170901 & 0.0154 & 0.2470 & 0.0380 \\
\hline 20171003 & 0.0085 & 0.0190 & 0.0410 \\
\hline 20171101 & 0.0055 & 0.0210 & 0.0420 \\
\hline 20171201 & 0.0040 & 0.0320 & 0.0170 \\
\hline
\end{tabular}

Figure 21: Power Variation down at monthly intervals along with support vector machine regression and linear regression equilibrium values based on three and six months of past parameter estimates. 

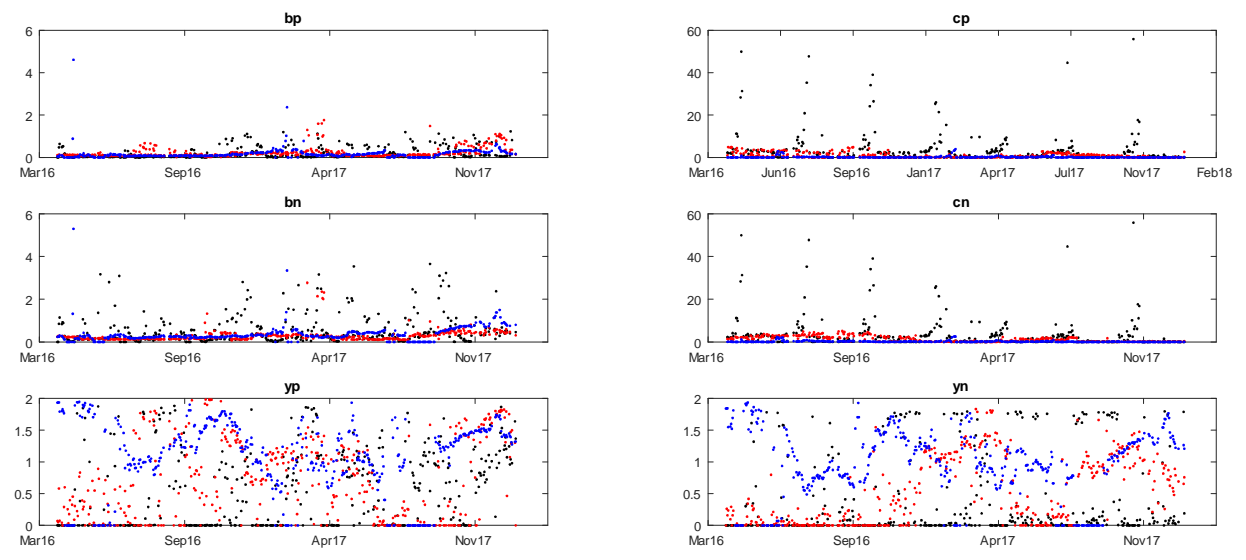

Figure 22: Power Variation risk neutral parameter Values in Black and the support vector machine regression finite variation equilibrium values in red.

steps that permit markets to react to each other. Portfolio and diversification considerations are then important as devices for controlling or managing the risk. Over short horizons risk exposure is being limited one asset at a time by reducing the holding period and portfolio considerations are less important. The correlations may also be reduced at the lower horizons. Many trading strategies with short holding periods seek to bias the gain probability in ones direction slightly to then benefit from doing a very large number of such trades. The assessment of the gain probability is done one asset at a time with little attention to portfolio considerations. Sometimes, as in pairs trading, one may consider a groups of assets taken together to seek a gain probability for a particular portfolio position and the attention shifts to the portfolio return distribution and we could add equilibrium portfolio returns distributions to our collection of equilibrium return distributions being studied. To some extent this is already present in the equilibrium distribution for an index or an exchange traded fund.

What collections of equilibrium return distributions do we expect to encounter in financial markets is our question. The question may be asked of both the physical return distributions and the risk neutral ones. For tractability we condition on fixed parametric classes of distributions that are here taken to be the bilateral gamma and power variation models already reported on. For each such distribution one may evaluate an arbitrage consistent performance measure. Here we employ the acceptability index introduced in Cherny and Madan (2009). The acceptability index is infinite for an arbitrage. It is defined by an increasing family $\mathcal{D}_{\gamma}$ of test probability measures $Q \in \mathcal{D}_{\gamma}$ with a random variable $X$ being acceptable at level $\gamma$ just if

$$
E^{Q}[X] \geq 0, \text { for all } Q \in \mathcal{D}_{\gamma} .
$$




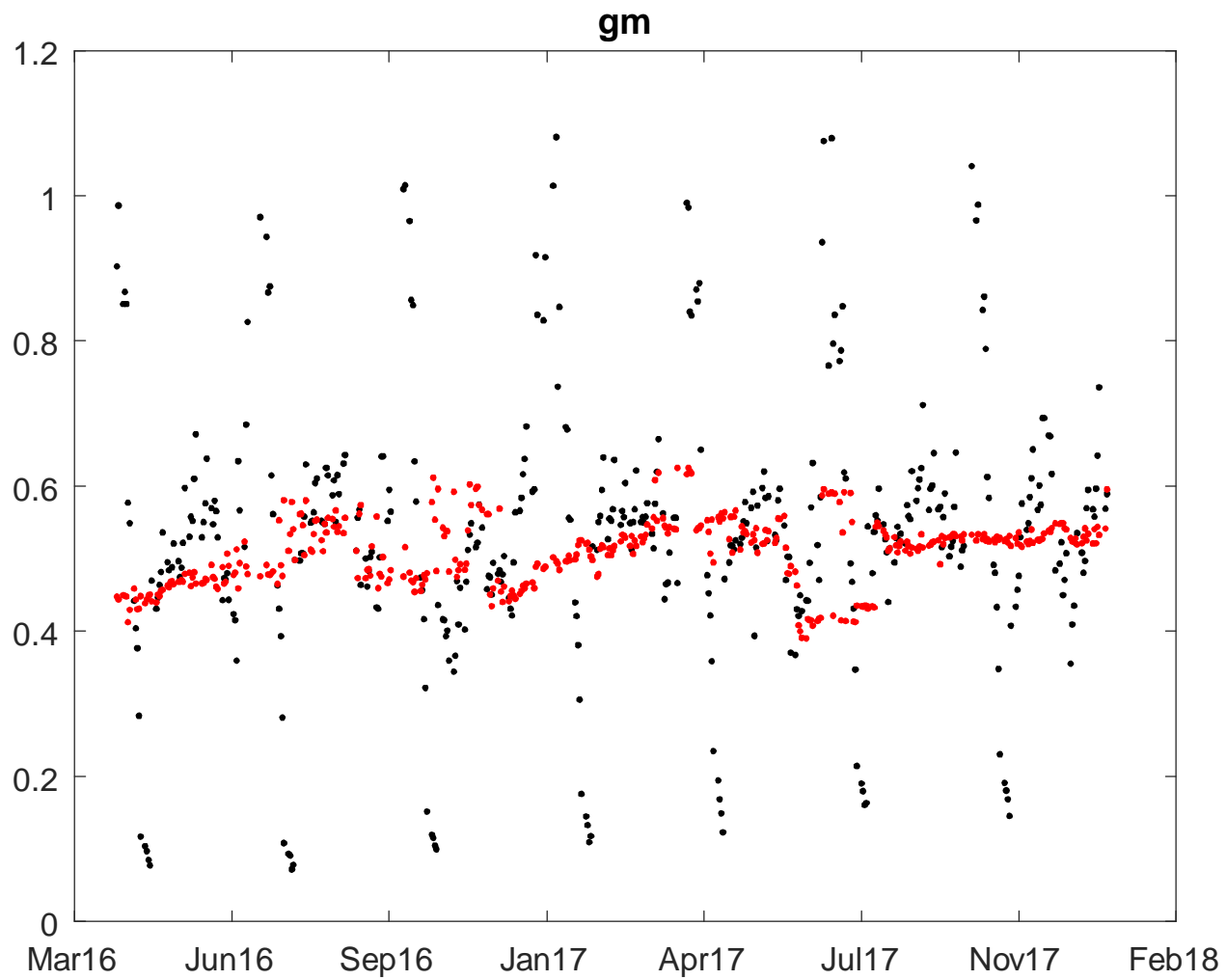

Figure 23: The data and equilibrium time scaling parameter values for amzn. 
The acceptability index is the highest level $\gamma^{*}(X)$ such that $X$ is acceptable for all $\gamma \leq \gamma^{*}$. The family of test measures increases towards the set of all measures and only an arbitrage is acceptable at all levels $\gamma>0$.

For an increasing sequence of concave distribution functions $\Psi^{\gamma}(u), 0 \leq u \leq$ 1, Cherny and Madan (2009) and Madan, Pistorius and Stadje (2017) introduced for a base probability measure $P$, the sets

$$
\mathcal{D}_{\gamma}=\left\{Q \mid Q(A) \leq \Psi^{\gamma}(P(A)), \text { for all } A\right\}
$$

for which acceptability at level $\gamma$ for a distribution function $F_{X}(x)$ reduces to a positive distorted expectation or

$$
\mathcal{E}^{\gamma}(X)=\int_{-\infty}^{\infty} x d \Psi^{\gamma}\left(F_{X}(x)\right) \geq 0 .
$$

The particular concave distortion we employ is the distortion termed minmaxvar in Cherny and Madan (2009) where we have

$$
\Psi^{\gamma}(u)=1-\left(1-u^{\frac{1}{1+\gamma}}\right)^{1+\gamma} .
$$

The acceptability index based on the distortion minmaxvar is the highest stress level $\gamma$ such that the distorted expectation $\mathcal{E}^{\gamma}(X)(2)$ is nonnegative. We anticipate that expected returns for equilibrium retrun distributions observed in the market are positive. As a consequence the acceptability indices for these distributions will be positive. However, we do not expect the acceptability indices of equilibrium return distributions observed in markets to get to high or be anywhere near an arbitrage.

For each of our 50 underliers we evaluated for each of 438 days the acceptability index for the equilibrium return distribution in the bilateral gamma class. We thus obtained a 438 by 50 matrix of acceptability indices. For each day we evaluated the quartiles across the 50 tickers of the equilibrium acceptability indices. Figure 24 presents these quartiles over time.

We observe that over this period the acceptability indices have risen from a median value 0.1 towards a median near 0.25 . The interquartile range near the end of the data period is between 0.15 and 0.3 .

The acceptability index of a return distribution has implications for the level of expected returns and how they are related to the risk. Furthermore there are implications for how this risk is to be measured. Consider the distorted expectation $\mathcal{E}^{\gamma}(X)$. The distorted expectation by construction is

$$
\mathcal{E}^{\gamma}(X)=\inf _{Q \in \mathcal{D}_{\gamma}} E^{Q}[X]
$$

It follows that

$$
\mathcal{E}^{\gamma}(X)=E^{P}[X]+\mathcal{E}^{\gamma}\left(X-E^{P}[X]\right) .
$$

We may then write the distorted expectation in the form of reward less risk by noting that

$$
\begin{aligned}
\mathcal{E}^{\gamma}(X) & =E^{P}[X]-\left(-\mathcal{E}^{\gamma}\left(-\left(E^{P}[X]-X\right)\right)\right) \\
& =E^{P}[X]-\sup _{Q \in \mathcal{D}_{\gamma}}\left(E^{P}[X]-X\right)
\end{aligned}
$$




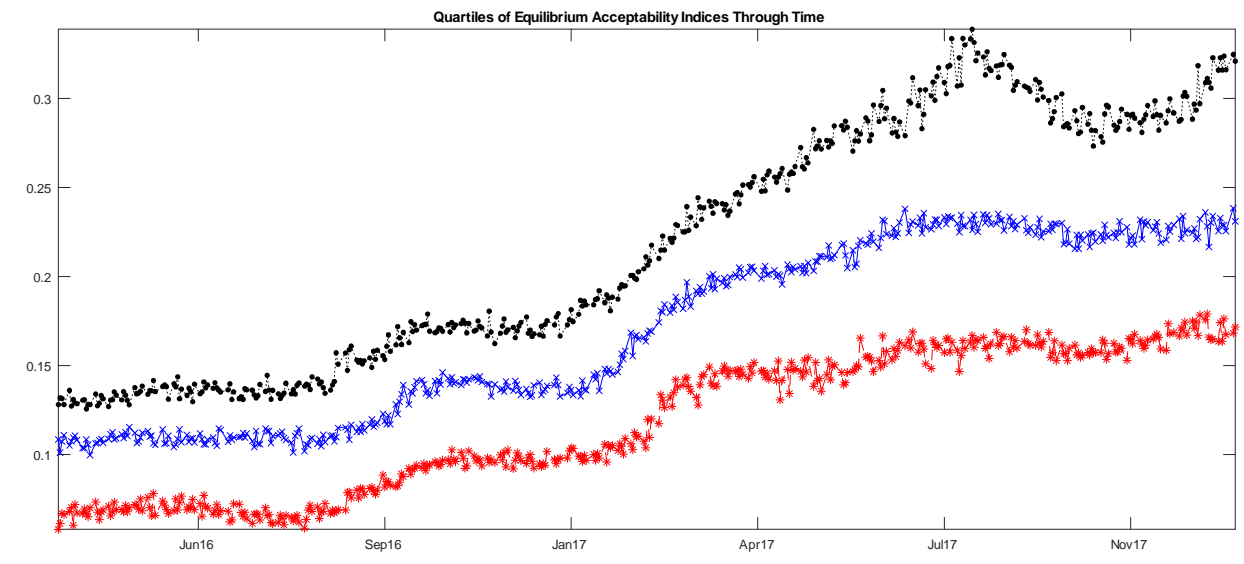

Figure 24: Quartiles for the equilibrium acceptability indices.

The risk is now

$$
\mathcal{R}_{\gamma}(X)=\sup _{Q \in \mathcal{D}_{\gamma}}\left(E^{P}[X]-X\right) .
$$

From the perspective of two price economies this risk is the ask price for the negated and centered random variable. Basically the centered random variable has no reward and is just risk that can be eliminated by buying its negative at the cost of the ask price for it. Hence the ask price for the negated centered random variable is the financial risk.

At the level of the acceptability index $\gamma^{*}$ it is the case that $\mathcal{E}^{\gamma^{*}}(X)=0$ and hence we have that

$$
E^{P}[X]=\mathcal{R}_{\gamma^{*}}(X) .
$$

This is a distributionally based asset pricing equation where required returns are equal to the risk as measured by the ask price for the negated and centered random variate. One may hypothesize that each day there exists a market target $\gamma_{t}^{*}$ that best explains the cross section of expected returns as this measure of risk for the said return. The level of $\gamma_{t}^{*}$ may be estimated from the cross section of equilibrium return distributions for the day.

For the equilibrium return distributions in the bilateral gamma class constructed using support vector machine regressions applied to three months of past data we estimate the cross sectional relation

$$
\mu_{i t}=\mathcal{R}_{\gamma_{t}^{*}}\left(R_{i t}\right)+\varepsilon_{i t}
$$

where $\mu_{i t}$ is the mean return on the $i^{t h}$ equilibrium return distribution on day $t$ and $R_{i t}$ is the $i^{t h}$ bilateral gamma distributed on day $t$. The estimation was conducted by least squares. Figure 25 presents the time series for $\gamma_{t}^{*}$.

We observe that the stress level consistent with the cross sectional asset pricing equation has been increasing over the period but was generally below 


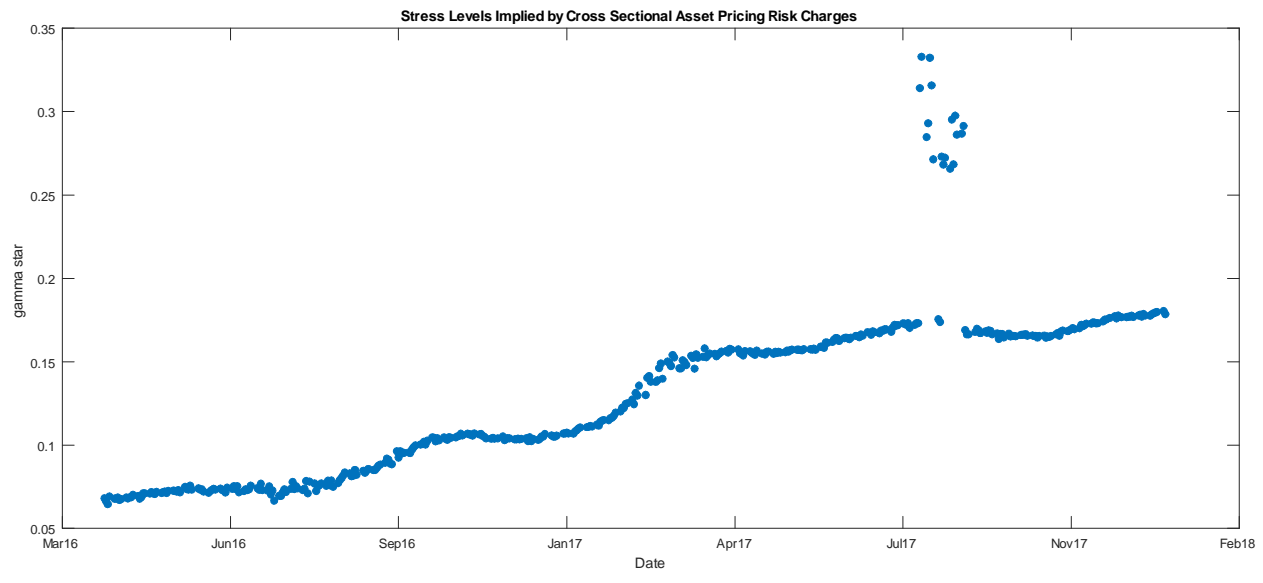

Figure 25: Stress level implied by cross sectional asset pricing model based on ask price for negated centered risk.

the median level. We regressed the mean returns cross sectionally on the risk charges $\mathcal{R}_{\gamma_{t}^{*}}\left(R_{i t}\right)$ to test for a unit slope and a zero intercept. Both these hypotheses were rejected. Figures 26 and 27 present the associated t-statistics.

Figure 28 presents the t-statistics for a zero slope. The zero slope is rejected in the latter period.

\section{Excitation and Cross Excitation}

The model with excitation and cross excitation requires a large number of ordinary differential equations to be solved with the output that then has to be sent for Fourier inversion. The estimation takes a few hours so it was done for 8 days on the first four underliers. Figure 29 presents the results for the time series data. There is evidence of some excitation but little cross excitation. The results for the risk neutral estimation are presented in Figures 8 and 8 . Figure 8 presents the dates and underliers for which the calibration converged to an absolute percentage error below $10 \%$, along with the fit statistics and the number of options employed with maturities below a month. Figure 8 presents the parameter estimates for the power variation model enhanced to allow for excitation and cross excitation. We observe that risk neutrally speed and scale are higher for the down moves as is the level of power variation. Also the down side has greater excitation and cross excitation. 


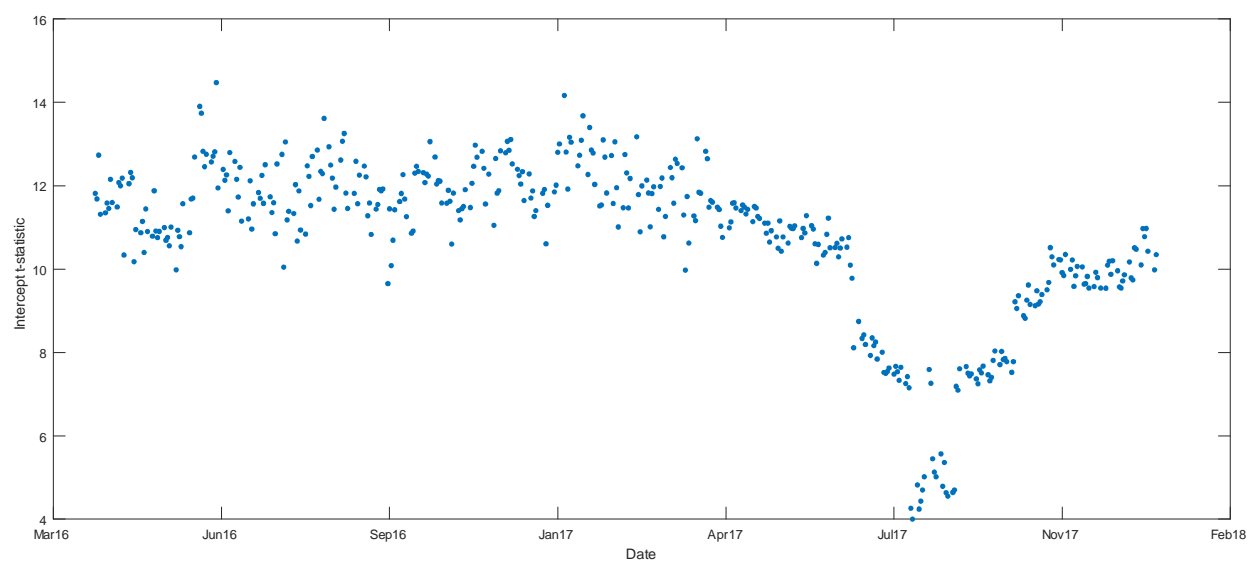

Figure 26: t-statistics for a zero intercept cross sectionally each day.

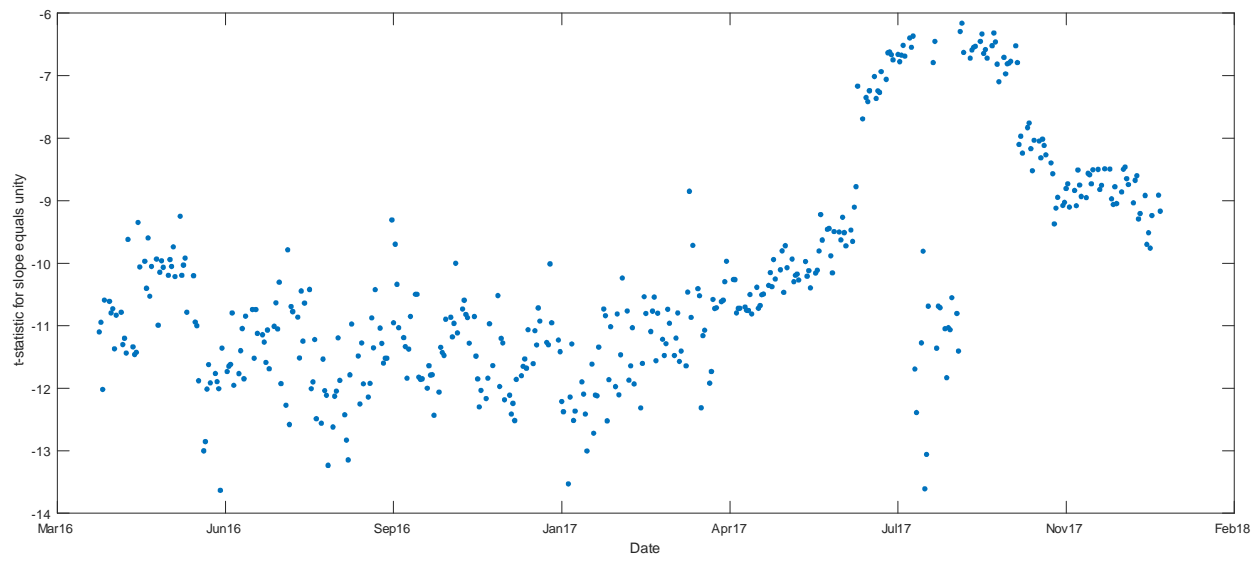

Figure 27: t-statistics for a unit slope coefficient. 


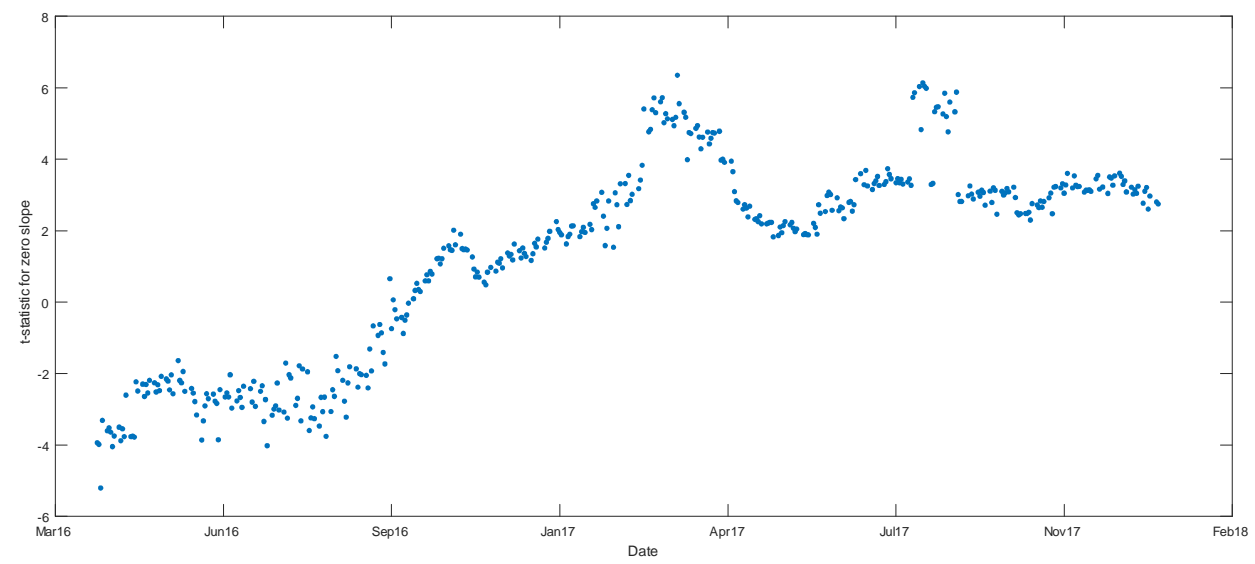

Figure 28: t-statistic for a zero slope.

\section{Conclusion}

Asset return distributions are taken to belong to the class of pure jump limit laws. Asymmetries between upward and downward motions are identified and documented. The return distributions are then modeled by self decomposable parametric laws where all the parameters are seen to be continuously responding to each other. Fixed points of the response functions define equilibrium distributions. The distributions that can arise in practice are seen to be constrained by the level of return acceptability they may attain. As a consequence expected returns reflect risk charges embedded in the ask price for the centered and negated return. The asymmetries studied include differences in scale, speed, power variation, excitation and cross excitation. 


\begin{tabular}{|c|c|c|c|c|c|c|c|c|c|c|c|}
\hline & \multicolumn{7}{|c|}{ Power Variation with Excitation and Cross Excitation } & \multirow[b]{2}{*}{$\mathrm{dp}$} & \multirow[b]{2}{*}{$\mathrm{dn}$} & \multirow[b]{2}{*}{ ep } & \multirow[b]{2}{*}{ en } \\
\hline & dates & $\mathrm{bp}$ & $\mathrm{cp}$ & bn & $\mathrm{cn}$ & yp & $\mathrm{yn}$ & & & & \\
\hline & 20160104 & 37.1824 & 2.1246 & 64.7113 & 0.7162 & 0.0106 & 0.0007 & 0.1257 & 0.2702 & 0.0631 & 0.1289 \\
\hline & 20160405 & 38.3210 & 1.9392 & 68.6987 & 0.7202 & 0.0255 & 0.0031 & 0.1089 & 0.1958 & 0.0817 & 0.1168 \\
\hline & 20160705 & 37.4567 & 1.9662 & 69.2027 & 0.6848 & 0.0242 & 0.0025 & 0.1106 & 0.2013 & 0.0778 & 0.1191 \\
\hline \multirow[t]{9}{*}{ spy } & 20161003 & 37.0723 & 2.0638 & 74.3142 & 0.5970 & 0.0120 & 0.0006 & 0.1387 & 0.2827 & 0.0650 & 0.1279 \\
\hline & 20170103 & 36.6645 & 1.9789 & 71.8798 & 0.5848 & 0.0173 & 0.0009 & 0.1300 & 0.2579 & 0.0692 & 0.1223 \\
\hline & 20170404 & 37.8021 & 1.8319 & 71.0658 & 0.5267 & 0.0178 & 0.0013 & 0.1298 & 0.2625 & 0.0648 & 0.1307 \\
\hline & 20170705 & 49.7614 & 1.1334 & 2.7343 & 1.4442 & 0.0289 & 0.0360 & 0.1050 & 0.1017 & 0.0987 & 0.1031 \\
\hline & 20171003 & 37.4607 & 1.5181 & 65.7003 & 0.4269 & 0.0359 & 0.0056 & 0.1026 & 0.1970 & 0.0726 & 0.1259 \\
\hline & 20160104 & 7.3047 & 16.4973 & 21.8410 & 14.4071 & 0.1383 & 0.0054 & 0.0524 & 0.1531 & 0.0783 & 0.1151 \\
\hline & 20160405 & 27.1545 & 4.8622 & 55.3686 & 2.1844 & 0.0233 & 0.0001 & 0.6245 & 0.2217 & 1.0847 & 0.2910 \\
\hline & 20160705 & 25.9480 & 4.9156 & 54.6635 & 2.2065 & 0.0293 & 0.0002 & 0.2291 & 0.8613 & 0.0283 & 0.0753 \\
\hline & 20161003 & 27.0693 & 4.5928 & 59.6226 & 1.8808 & 0.0254 & 0.0001 & 0.3722 & 1.5110 & 0.0084 & 0.0510 \\
\hline \multirow[t]{7}{*}{ iwm } & 20170103 & 25.6747 & 5.1147 & 57.0495 & 2.0686 & 0.0238 & 0.0000 & 1.4511 & 1.1719 & 1.5395 & 3.0996 \\
\hline & 20170404 & 25.5070 & 5.1100 & 50.3895 & 2.5278 & 0.0331 & 0.0004 & 0.2415 & 0.8201 & 0.0209 & 0.0730 \\
\hline & 20170705 & 29.7803 & 3.9268 & 53.5041 & 2.0816 & 0.0348 & 0.0021 & 0.0993 & 0.2461 & 0.0722 & 0.1086 \\
\hline & 20171003 & 30.8578 & 3.6658 & 56.4464 & 1.8377 & 0.0318 & 0.0020 & 0.1029 & 0.2334 & 0.0751 & 0.1091 \\
\hline & 20160104 & 41.2941 & 2.2150 & 77.4032 & 0.7677 & 0.0111 & 0.0005 & 0.1616 & 0.2845 & 0.0740 & 0.1189 \\
\hline & 20160405 & 45.7831 & 1.9302 & 85.3603 & 0.6889 & 0.0159 & 0.0028 & 0.1507 & 0.2259 & 0.0853 & 0.1140 \\
\hline & 20160705 & 46.1286 & 1.8821 & 86.7764 & 0.6357 & 0.0145 & 0.0019 & 0.1486 & 0.2374 & 0.0830 & 0.1179 \\
\hline \multirow[t]{8}{*}{ q११ } & 20161003 & 44.7814 & 1.8891 & 89.6698 & 0.5889 & 0.0148 & 0.0010 & 0.1499 & 0.2670 & 0.0794 & 0.1199 \\
\hline & 20170103 & 43.2314 & 1.9415 & 88.6391 & 0.5870 & 0.0145 & 0.0007 & 0.1456 & 0.2884 & 0.0748 & 0.1234 \\
\hline & 20170404 & 42.6551 & 1.9306 & 92.5252 & 0.5023 & 0.0099 & 0.0002 & 0.1307 & 0.3498 & 0.0495 & 0.1443 \\
\hline & 20170705 & 44.2784 & 1.7560 & 95.6202 & 0.4429 & 0.0136 & 0.0003 & 0.1784 & 0.3504 & 0.0601 & 0.1324 \\
\hline & 20171003 & 44.1175 & 1.7877 & 95.0997 & 0.4352 & 0.0099 & 0.0002 & 0.1819 & 0.3580 & 0.0542 & 0.1376 \\
\hline & 20160104 & 78.4674 & 1.9515 & 92.1684 & 1.3397 & 0.0176 & 0.0121 & 0.1257 & 0.1596 & 0.0999 & 0.0979 \\
\hline & 20160405 & 80.7389 & 1.7919 & 99.4135 & 1.2155 & 0.0232 & 0.0147 & 0.1070 & 0.1414 & 0.1009 & 0.0983 \\
\hline & 20160705 & 73.1112 & 2.0850 & 94.5364 & 1.3371 & 0.0156 & 0.0097 & 0.1281 & 0.1814 & 0.0979 & 0.0996 \\
\hline \multirow[t]{5}{*}{ aapl } & 20161003 & 77.8730 & 1.8801 & 97.5428 & 1.2171 & 0.0151 & 0.0099 & 0.1318 & 0.1863 & 0.0952 & 0.1035 \\
\hline & 20170103 & 72.8947 & 1.8933 & 93.6449 & 1.1582 & 0.0183 & 0.0126 & 0.1252 & 0.1631 & 0.0988 & 0.1002 \\
\hline & 20170404 & 74.7961 & 1.7009 & 100.0083 & 0.9307 & 0.0145 & 0.0092 & 0.1422 & 0.1858 & 0.0976 & 0.1025 \\
\hline & 20170705 & 76.4239 & 1.5958 & 105.0884 & 0.8228 & 0.0117 & 0.0067 & 0.1569 & 0.2058 & 0.0973 & 0.1037 \\
\hline & 20171003 & 73.5527 & 1.6610 & 102.3910 & 0.8339 & 0.0070 & 0.0037 & 0.1811 & 0.2397 & 0.0985 & 0.1029 \\
\hline
\end{tabular}

Figure 29: Parameter estimates for power variation extended to include excitation and cross excitation. 


\begin{tabular}{|c|c|c|c|c|c|}
\hline \multicolumn{5}{|c|}{ Risk Neutral Results for PVECE } & \\
\hline dates & underlier & rmse & aae & ape & nop \\
\hline 20160104 & spy & 0.2999 & 0.2126 & 0.0788 & 1008 \\
\hline 20160405 & spy & 0.2144 & 0.1766 & 0.0631 & 1015 \\
\hline 20161003 & spy & 0.1721 & 0.1375 & 0.0579 & 1164 \\
\hline 20170404 & spy & 0.1866 & 0.1467 & 0.0672 & 1165 \\
\hline 20170404 & iwm & 0.1278 & 0.1055 & 0.0580 & 1114 \\
\hline 20160104 & iwm & 0.1647 & 0.1357 & 0.0670 & 781 \\
\hline 20171003 & iwm & 0.2106 & 0.1826 & 0.0935 & 989 \\
\hline
\end{tabular}

\begin{tabular}{|c|c|c|c|c|c|c|c|c|c|}
\hline \multirow[b]{2}{*}{$\mathrm{cp}$} & \multicolumn{5}{|c|}{ PVECE Risk Neutral Parameter estimates } & \multirow[b]{2}{*}{$d p$} & \multirow[b]{2}{*}{$\mathrm{dn}$} & \multirow[b]{2}{*}{ ep } & \multirow[b]{2}{*}{ en } \\
\hline & $\mathrm{cn}$ & bp & bn & yp & yn & & & & \\
\hline 0.1415 & 0.2969 & 0.0029 & 0.2076 & 0.0591 & 0.8900 & 0.0100 & 0.5338 & 0.0796 & 0.0252 \\
\hline 0.0165 & 0.0656 & 0.4614 & 0.4498 & 0.0582 & 1.1598 & 0.0422 & 0.1010 & 0.7245 & 0.0002 \\
\hline 0.0195 & 0.0954 & 0.5634 & 0.2290 & 0.0317 & 1.0564 & 0.0044 & 0.5630 & 0.9828 & 0.7648 \\
\hline 0.0022 & 0.0218 & 1.4900 & 0.6309 & 0.0258 & 1.3664 & 2.2335 & 0.1364 & 0.2215 & 1.2225 \\
\hline 0.9303 & 0.0770 & 0.0303 & 0.3140 & 0.0731 & 1.2614 & 0.0539 & 0.1093 & 3.1071 & 0.6385 \\
\hline 0.0010 & 0.0226 & 0.0076 & 7.8068 & 0.0315 & 1.6293 & 0.0459 & 0.0302 & 0.3733 & 0.7681 \\
\hline 0.0004 & 0.0114 & 0.0121 & 15.8016 & 0.0267 & 1.6592 & 0.0399 & 0.0135 & 0.6610 & 1.1446 \\
\hline
\end{tabular}

\section{APPENDIX}

The ticker symbols for the underliers reported on are presented in Figure 30 


\begin{tabular}{|c|c|c|c|c|}
\hline \multicolumn{5}{|c|}{ Underlying Asset Ticker Symbols } \\
\hline spy & $\mathrm{msft}$ & wfc & $\mathrm{gm}$ & gild \\
\hline iwm & nflx & dis & v & twtr \\
\hline qqq & C & gs & bidu & wynn \\
\hline aapl & amzn & xIf & xom & pypl \\
\hline $\mathrm{fb}$ & eem & $\mathrm{t}$ & $\mathrm{cmg}$ & ge \\
\hline nvda & tsla & xle & $n d x$ & $\mathrm{CSCO}$ \\
\hline baba & intc & rut & $g d x$ & goog \\
\hline $\mathrm{mu}$ & xop & wmt & amd & xlu \\
\hline gld & ewz & jd & dia & hd \\
\hline bac & jpm & ba & googl & cmcsa \\
\hline
\end{tabular}

Figure 30: Ticker Symbols for the Assets reported on for both the physical returns distributions and their risk neutral counterparts.

\section{References}

[1] Bonanno, G., F. Lillo and R. N. Mantegna (2001), "High-frequency Crosscorrelation in a Set of Stocks," Quantitative Finance, 1, 96-104.

[2] Carr, P., H. Geman, D. Madan, D. and M. Yor (2002), "The fine structure of asset returns: An empirical investigation," Journal of Business, 75, 2, 305-332.

[3] Carr, P., Geman, H., Madan, D. B. and Yor, M: Self-Decomposability and Option Pricing. Mathematical Finance, 17, 31-57 (2007)

[4] Carr, P. and D. B. Madan, (2014), "Joint Modeling of VIX and SPX options at a single and common maturity with risk management applications," IIE Transactions, 46, 1125-1131.

[5] Cherny, A. and D. B. Madan (2009), "New Measures of Performance Evaluation," Review of Financial Studies, 22, 2571-2606.https://doi.org/10. 1093/rfs/hhn081

[6] Cochrane, J. H. (2001), Asset Pricing, Princeton University Press. Princeton, New Jersey.

[7] Duffie, D. (2001), Dynamic Asset Pricing Theory, 3rd Edition, Princeton University Press, Princeton, New Jersey.

[8] Duffie, D., D. Filipović and W. Schachermayer (2003), "Affine Processes and Applications to Finance," The Annals of Applied Probability, 13, 9841053. 
[9] Engle, R. F., E. Ghysels and B. Sohn (2008), "One the Economic Sources of Stock Market Volatility," Working Paper New York University, available at SSRN: https://ssrn.com/abstract=971310

[10] Epps, T. W. (1979), "Comevements in Stock Prices in the Very Short Run," Journal of the American Statistical Association, 74, 291-298.

[11] Khintchine, A. Y. (1938), "Limit laws of sums of independent random variables," ONTI, Moscow, (Russian).

[12] Lévy, P. (1937), Théorie de l'Addition des Variables Aléatoires, GauthierVillars, Paris.

[13] Lintner, J. (1965), "The Valuation of Risk Assets and the Selection of Risky Investments in Stock Portfolios and Capital Budgets," Review of Economics and Statistics, 47, 13-37.

[14] Madan D., P. Carr and E. Chang (1998), "The variance gamma process and option pricing," European Finance Review, 2,79-105.

[15] Madan, D., M. Pistorius and M. Stadje (2017), "On dynamic spectral risk measures, a limit theorem and optimal portfolio allocation, Finance and Stochastics, 21, 1073-1102.

[16] Madan, D. B. and W. Schoutens (2018), "Self Similarity in Long Horizon Returns," available at SSRN: https://ssrn.com/abstract=3102406

[17] Madan, D. B., W. Schoutens and K. Wang (2017), "Measuring and Monitoring the Efficiency of Markets," International Journal and Theoretical and Applied Finance, 20,

[18] Madan D. B. and E. Seneta (1990), "The variance gamma (VG) model for share market returns," Journal of Business, 63, 511-524.

[19] Madan, D. B. and K. Wang (2017), "Asymmetries in Financial Returns," International Journal of Financial Engineering, https://doi.org/10. 1142/S2424786317500451.

[20] Mossin, J. (1966), "Equilibrium in a Capital Asset Market," Econometrica, 35, $768-783$.

[21] Ross, S.A. (1976), "Arbitrage Theory of Capital Asset Pricing," Journal of Economic Theory, 13, 341-360.

[22] Skiadas, C. (2009), Asset Pricing Theory, Princeton University Press, Princeton, New Jersey.

[23] Sato, K. (1991), "Self similar processes with independent increments," Probability Theory and Related Fields, 89, 285-300. 
[24] Sato, K. (1999), Lévy processes and Infinitely Divisible Distributions, Cambridge University Press, Cambridge.

[25] Schwert, G. W. (1989), "Why Does Stock Market Volatility Change Over Time?," Journal of Finance, 44, 1207-1239.

[26] Sharpe, W.F. (1964), "Capital Asset Prices: A theory of Market Equilibrium under Conditions of Risk," Journal of Finance, 19, 425-442.

[27] Weitzman, M. (2007), "Subjective Expectations and Asset-Return Puzzles," American Economic Review, February,1102-1130. 